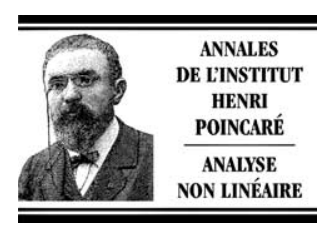

www.elsevier.com/locate/anihpc

\title{
Multitransition kinks and pulses for fourth order equations with a bistable nonlinearity
}

\section{Denis Bonheure}

Université catholique de Louvain, institut de mathématique pure et appliquée, chemin du Cyclotron, 21348 Louvain-la-neuve, Belgium Received 22 October 2002; received in revised form 10 March 2003; accepted 31 March 2003

Available online 25 September 2003

\begin{abstract}
We study the existence of stationary solutions of a class of diffusion equations related to the so-called extended FisherKolmogorov equation and the Swift-Hohenberg equation. We prove the existence of mu ltitransition kinks and pulses. These solutions are obtained as local minima of the associated functional. For the Swift-Hohenberg equation, our result partially proves a numerical conjecture.

(C) 2004 L'Association Publications de l'Institut Henri Poincaré. Published by Elsevier B.V. All rights reserved
\end{abstract}

\section{Résumé}

Nous étudions l'existence de solutions stationnaires d'une classe d'équations de diffusion incluant l'équation de FisherKolmogorov généralisée et l'équation de Swift-Hohenberg. Nous démontrons l'existence de solutions hétéroclines et homoclines à transitions multiples. Ces solutions sont des minima locaux de la fonctionnelle associée. Nos résultats couvrent partiellement une conjecture numérique concernant l'équation de Swift-Hohenberg.

(C) 2004 L'Association Publications de l'Institut Henri Poincaré. Published by Elsevier B.V. All rights reserved

MSC: $34 \mathrm{C} 37 ; 37 \mathrm{~J} 45$

Keywords: Multitransition heteroclinics and homoclinics; Swift-Hohenberg equation; Minimization; Saddle-focus equilibrium

\section{Introduction}

This paper is concerned with the study of particular stationary solutions of a class of semilinear diffusion equations of the form

$$
\frac{\partial u}{\partial t}+\frac{\partial^{4} u}{\partial x^{4}}-\beta \frac{\partial^{2} u}{\partial x^{2}}+u^{3}-u=0
$$

where $\beta$ is a real parameter. This equation is a model in many physical, chemical or biological systems. When $\beta>0$, it is related to the so-called Extended Fisher-Kolmogorov equation which was proposed in 1988 by Dee

E-mail address: bonheure@anma.ucl.ac.be (D. Bonheure).

0294-1449/\$ - see front matter C 2004 L'Association Publications de l'Institut Henri Poincaré. Published by Elsevier B.V. All rights reserved doi:10.1016/j.anihpc.2003.03.001 
and van Saarloos [5] as an higher-order model equation for physical systems that are bistable. By a scaling in the variable $x$, Eq. (1) can be written as

$$
\frac{\partial u}{\partial t}+\gamma \frac{\partial^{4} u}{\partial x^{4}}-\frac{\partial^{2} u}{\partial x^{2}}+u^{3}-u=0
$$

where the positive parameter $\gamma$ is related to $\beta$ by the formula $\beta=1 / \sqrt{\gamma}$. When $\gamma=0$ in (2), we recognize the well known Fisher-Kolmogorov equation, sometimes also called the Allen-Cahn equation, originally introduced in 1937 [10] as a model for studying biological populations. The term bistable indicates that the Fisher-Kolmogorov equation and its extended version have two uniform stable states $u(x)= \pm 1$ separated by a third uniform state $u(x)=0$ which is unstable, see [5].

When $\beta$ is negative, Eq. (1) is related to the Swift-Hohenberg equation

$$
\frac{\partial u}{\partial t}-\kappa u+\left(1+\frac{\partial^{2}}{\partial x^{2}}\right)^{2} u+u^{3}=0,
$$

where $\kappa \in \mathbb{R}$. This equation was proposed by Swift and Hohenberg [16] as a model in the study of Rayleigh-Bénard convection. When $\kappa>1$, this equation can be transformed into

$$
\frac{\partial u}{\partial t}+(\kappa-1)^{3 / 2}\left[\frac{\partial^{4} u}{\partial x^{4}}-\beta \frac{\partial^{2} u}{\partial x^{2}}+u^{3}-u\right]=0
$$

with $\beta=-2 / \sqrt{\kappa-1}$.

For these model equations, a question of great interest is the existence of phase transitions, i.e. solutions that spatially connect two uniform states. When looking at time-independent solutions, we are lead to the following autonomous equation

$$
u^{\prime \prime \prime \prime}-\beta u^{\prime \prime}+u^{3}-u=0 .
$$

Heteroclinic solutions of (4) (or kinks) connecting -1 and +1 in the phase-space, i.e. solutions that satisfy the following conditions

$$
\lim _{x \rightarrow \pm \infty}\left(u(x), u^{\prime}(x), u^{\prime \prime}(x), u^{\prime \prime \prime}(x)\right)=( \pm 1,0,0,0)
$$

are then stationary solutions of (1) connecting the two uniform states -1 and +1 . Of course, we can also consider heteroclinics from +1 to -1 .

Nonlinear Schrödinger equations are also related to the model equation (4). When assuming harmonic spatial dependence, i.e. $v(x, t)=u(t) \mathrm{e}^{i k x}$ for some $k \in \mathbb{R}$, the solutions of the Schrödinger equation

$$
\mathrm{i} \frac{\partial v}{\partial x}+\frac{\partial^{2} v}{\partial t^{2}}-\frac{\partial^{4} v}{\partial t^{4}}+|v|^{2} v=0
$$

solve, after scaling, Eq. (4) with $\beta=1 / \sqrt{k}$ and a question of interest in optic is the existence of pulse propagation. For Eq. (4), this amounts to study the existence of homoclinic solutions (or pulses), i.e. solutions of (4) with the property

$$
\lim _{x \rightarrow \pm \infty}\left(u(x), u^{\prime}(x), u^{\prime \prime}(x), u^{\prime \prime \prime}(x)\right)=(1,0,0,0) \quad(\text { or }(-1,0,0,0))
$$

The study of Eq. (4) for positive values of the parameter $\beta$ goes back at least to Peletier and Troy in [11,12] where they proved among other things, the existence of kinks for all $\beta>0$. When $\beta \in[\sqrt{8}, \infty)$, van den Berg [18] proved that the bounded solutions of (4) behave like the bounded solutions of the stationary Fisher-Kolmogorov equation

$$
-u^{\prime \prime}+u^{3}-u=0
$$


This implies that there exist two kinks (up to translations), one monotone increasing from -1 to +1 and its symmetric, while there are no pulses. When $\beta \in[0, \sqrt{8})$, the set of kinks and pulses is much more rich. For this range of $\beta$, kinks and pulses cannot be monotone anymore as at $\beta=\sqrt{8}$, both equilibria \pm 1 bifurcate from saddle-nodes to saddle-foci. The linearization of (4) around the equilibria then shows that the solutions oscillate when they are close to \pm 1 with small derivatives up to the third order. As $\beta$ becomes smaller than $\sqrt{8}$, infinitely many kinks and pulses appear. Peletier and Troy [12] proved the existence of two infinite sequences of both kinks and pulses. The two sequences of kinks consist of odd kinks having $2 n+1$ zeros and differ in the amplitude of the oscillations. The pulses are even with $2 n$ zeros. Again, the two sequences can be distinguished according to the amplitude of the oscillations. Other families of kinks and pulses were shown to exist [8,9]. Basically, these solutions can be distinguished by the number of jumps from -1 to +1 and the oscillations around these equilibria in between the jumps. The complex structure of these solutions can be quantified by defining homotopy classes, see [8].

Different methods have been used to deal with Eq. (4). Peletier and Troy introduced in [11,12] a topological shooting method that can be used to track kinks and pulses as well as periodic solutions. In [14], it is shown that kinks and periodic solutions can be obtained using variational arguments. For instance, if $\beta \geqslant 0$ the functional

$$
\mathcal{J}_{\beta}^{*}(u)=\int_{-\infty}^{+\infty}\left[\frac{1}{2}\left[\left(u^{\prime \prime 2}\right)+\beta u^{\prime 2}\right]+\frac{1}{4}\left(u^{2}-1\right)^{2}\right] \mathrm{d} x
$$

has a minimum in the function space $\mathcal{H}=\chi+H^{2}(\mathbb{R})$ where $\chi$ is a $C^{\infty}$ function that satisfies $\chi(x)=-1$ for $x \leqslant-1$ and $\chi(x)=1$ for $x \geqslant 1$. When $\beta \geqslant \sqrt{8}$, this minimizer is the unique heteroclinic connection from -1 to +1 , while for $\beta<\sqrt{8}$ it is called the principal heteroclinic as it only has one zero.

The dynamics of Eq. (4) with $\beta<0$ is much less understood than the one of the Extended Fisher-Kolmogorov equation. Numerical experiments [17] suggest that a large variety of those solutions found for $\beta$ positive still exist for a certain range of negative values of $\beta$. A study of periodic solutions was recently presented in [19]. For kinks and pulses, the limitation of the shooting method of Peletier and Troy was pointed out in [20] while the term $\beta u^{\prime 2}$ in the functional $\mathcal{J}_{\beta}^{*}$ is very "bad" for minimization when $\beta<0$.

However recent applications of variational arguments were shown to be fruitful. Smets and van den Berg [15] have used a version of the mountain pass theorem to prove the existence of at least one homoclinic solution at each equilibria for almost every $\beta \in(-\sqrt{8}, 0)$. In [3], looking for instance at heteroclinic solutions, it is shown that minimization arguments can still be used for $\beta$ greater than some negative $\beta_{0}$ which can be characterized by

$$
\beta_{0}=\inf \left\{\beta<0 \mid \inf _{\mathcal{H}} \mathcal{J}_{\beta}^{*} \geqslant 0\right\}
$$

while $\mathcal{J}_{\beta}^{*}$ is unbounded from below beyond $\beta_{0}$. Numerical computations [17] indicate that $\beta_{0}$ is close to -0.9 . The solution found by minimization still corresponds to the principal kink.

In this paper, we obtain multitransition kinks and pulses for $\beta \geqslant \beta_{0}$. By a transition, we mean a passage from -1 to 1 or reversely. Actually, we consider the more general functional

$$
\mathcal{F}^{*}(u)=\int_{-\infty}^{+\infty}\left[\frac{1}{2}\left(u^{\prime \prime 2}+g(u) u^{\prime 2}\right)+f(u)\right] \mathrm{d} x
$$

whose Euler-Lagrange equation is given by

$$
u^{\prime \prime \prime \prime}-g(u) u^{\prime \prime}-\frac{1}{2} g^{\prime}(u) u^{\prime 2}+f^{\prime}(u)=0 .
$$

Here we assume that the function $f$ is a symmetric double-well potential with bottoms at \pm 1 and $g$ is an even function which is not necessarily constant. The interest of considering a nonconstant function $g$ can be checked 
in [6]. Functionals of the form (8) were already considered in [2,3,7] with either a double-well or a triple-well potential $f$ and a function $g$ that can take either sign.

Let us first look at heteroclinic connections of (9). Since we assume that $f$ and $g$ are even, we can restrict our attention to odd solutions. Indeed, given any function $u \in \mathcal{H}$, it is easy to build an odd function $u^{*} \in \mathcal{H}$ having smaller action than $u$, see [9]. We thus look at the critical points of the functional

$$
\mathcal{F}(u)=\int_{0}^{+\infty}\left[\frac{1}{2}\left(u^{\prime \prime 2}+g(u) u^{\prime 2}\right)+f(u)\right] \mathrm{d} x
$$

in the space

$$
\mathcal{E}=\left\{u \mid u-1 \in H^{2}\left(\mathbb{R}^{+}\right), u(0)=0\right\} .
$$

If $\mathcal{F}$ has a minimizer $u$, an easy computation shows that $u^{\prime \prime}(0)=0$. Extending then $u$ on $\mathbb{R}$ by

$$
u^{*}(x)= \begin{cases}-u(-x) & \text { if } x<0 \\ u(x) & \text { if } x \geqslant 0\end{cases}
$$

we obtain an odd solution of (9). Also, it is easy to check that conditions (5) are fulfilled.

As we have already mentioned in the case of the model Eq. (4), we expect multitransition solutions when the equilibria \pm 1 are saddle-foci, i.e. when $g(1)^{2}<4 f^{\prime \prime}(1)$. We obtain these solutions as local minima of the functional $\mathcal{F}$ in appropriate subsets of $\mathcal{E}$. Basically, these subsets correspond to classes of functions having the desired number of transitions. We define for each $n \geqslant 0$, the subset $\mathcal{E}_{n} \subset \mathcal{E}$ consisting of functions whose odd extensions on $\mathbb{R}$ make $2 n+1$ transitions. More precisely, a function $u \in \mathcal{E}$ belongs to the subclass $\mathcal{E}_{n}$ if there exist $0=x_{0}<x_{1}<\cdots<x_{n}<x_{n+1}=\infty$ such that

$$
\begin{aligned}
& u(x)(-1)^{i+n}>0 \text { for } x \in\left(x_{i}, x_{i+1}\right), \\
& \max _{\left(x_{i}, x_{i+1}\right)} u(x)(-1)^{i+n}>1 .
\end{aligned}
$$

We prove that $\mathcal{F}$ has a local minimum in each of these subspaces in the two following situations.

Theorem 1. Let $f$ and $g \in C^{2}(\mathbb{R})$ be even functions such that $f(1)=0$ and assume that for some $k>0$, $\beta \in[0, \sqrt{8 k})$ and all $u \geqslant 0$,

$$
f(u) \geqslant k(u-1)^{2} \text { and } g(u) \geqslant-\beta
$$

and $g(1)^{2}<4 f^{\prime \prime}(1)$. Assume further that

$$
\inf _{\mathcal{E}} \mathcal{F}>-\infty
$$

where $\mathcal{F}: \mathcal{E} \rightarrow \mathbb{R}$ is defined by (10). Then, for every $n \in \mathbb{N}, \mathcal{F}$ has a local minimizer $u_{n}$ in the subspace $\mathcal{E}_{n}$. Moreover, the odd extension of $u_{n}$ on $\mathbb{R}$ is an heteroclinic solution of (9) having exactly $2 n+1$ zeros.

Theorem 2. Let $f$ and $g \in C^{2}(\mathbb{R})$ be even functions such that $f(1)=f^{\prime}(1)=0$ and for some function $\tilde{g} \in C(\mathbb{R})$ and some $k<1$,

$$
g(u) \geqslant \tilde{g}(u), \quad|\widetilde{G}(u)| \leqslant k \sqrt{8 f(u)}, \quad \forall u \in \mathbb{R},
$$

where $\widetilde{G}(u):=\int_{0}^{u} \tilde{g}(s) \mathrm{d} s$. Assume moreover that $g(1)^{2}<4 f^{\prime \prime}(1)$. Then the conclusion of Theorem 1 holds.

The case of a nonnegative function $g$, which is covered either by Theorem 1 and 2, is already contained in [8] where even more precise results are given. It is proved therein that each subset $\mathcal{E}_{n}$ can again be divided in smaller classes to obtain more kinks. In a subset $\mathcal{E}_{n}$, these solutions can be distinguished by the number of oscillations 
around \pm 1 in between the transitions. The solutions that we obtain in Theorems 1 and 2 correspond to those found in [8] in the particular case of two crossings with \pm 1 in each transition.

It is pointed out in [8] that the methods used therein require a nonnegative Lagrangian

$$
L\left(u, u^{\prime}, u^{\prime \prime}\right)=\frac{1}{2}\left(u^{\prime \prime 2}+g(u) u^{\prime 2}\right)+f(u)
$$

In this case, the action

$$
\mathcal{F}(u)=\int L\left(u, u^{\prime}, u^{\prime \prime}\right) \mathrm{d} x
$$

is of course nonnegative along admissible functions. Our results can be seen as a partial extension of the methods of [8] to Lagrangians that can take either sign. To deal with such Lagrangians, we only require an a priori lower bound on the action along admissible functions. This condition is explicitly stated in Theorem 1 while it is a consequence of assumption (14) in Theorem 2. This boundedness assumption on the functional might seem rather artificial. However, if $f$ and $g$ satisfy the assumptions of Theorem 1, it can be checked that $\mathcal{F}$ is bounded from below on $\mathcal{E}$ at least for $\beta>0$ small enough, see Lemma 4 in [3]. Hence, we can state the following proposition.

Proposition 3. Let $f \in C^{2}(\mathbb{R})$ be an even function such that $f(1)=0$ and for some $k>0, f(u) \geqslant k(u-1)^{2}$ for all $u \geqslant 0$. Then, there exists $\beta(f) \in(0, \sqrt{8 k})$ such that if $\beta \leqslant \beta(f)$,

$$
\inf _{u \in \mathcal{E}} \int_{0}^{+\infty}\left[\frac{1}{2}\left(u^{\prime \prime 2}-\beta u^{\prime 2}\right)+f(u)\right] \mathrm{d} x>-\infty .
$$

Moreover, if $g \in C^{2}(\mathbb{R})$ is even, satisfies $g(u) \geqslant-\beta(f)$ for $u \geqslant 0$ and $g(1)^{2}<4 f^{\prime \prime}(1)$, the conclusion of Theorem 1 holds.

It is interesting to notice that a lower bound on the functional implies in fact that the action of any admissible function is positive, see Section 4 and [3].

For a function $u \in \mathcal{E}_{n}$, we denote by $I_{i}$ the intervals $\left(x_{i}, x_{i+1}\right)$ for $i=0, \ldots, n$ where by convention $x_{0}=0$ and $x_{n+1}=\infty$. The main idea in the proof of Theorems 1 or 2 is to show the existence of a minimizing sequence $\left(u_{p}\right)_{p} \subset \mathcal{E}_{n}$ that has the following properties:

(a) there exists $I>0$ such that for all $u_{p},\left|I_{i}\right| \leqslant I$ for all $i=0, \ldots, n-1$;

(b) there exists $C>0$ such that for all $u_{p},\left\|u_{p}-1\right\|_{H^{2}} \leqslant C$.

These two properties are closely related as they prevent from a loss of compactness when extracting a weak converging subsequence.

The main tool for obtaining the estimates on the length of the intervals $I_{i}$ is the clipping procedure introduced in [8]. Also, the oscillatory behaviour of minimizers close to the equilibria \pm 1 (described in Section 2.2) is crucial in the construction of a minimizing sequence having the above properties.

Equivalent results can be obtained for homoclinic solutions. We show in Section 5 how to adapt the definition of the classes $\mathcal{E}_{n}$ to find even homoclinic connections. Of course, these classes have to be defined in another functional space that takes conditions (6) into account.

Sections 2 and 3 are devoted to preliminaries to the proof of Theorem 1. In Section 3, we construct a minimizing sequence with the properties (a) and (b) mentioned above. The proof of Theorem 1 and a sketch of the proof of Theorem 2 are given in Section 4.

Finally, it is worth mentioning that a good account on known results about Eq. (4) and related model equations can be found in the recent book of Peletier and Troy [13]. 


\section{Auxiliary results}

In this section, we prove some preliminary results that are used as main tools in the minimizing process.

\subsection{Inequalities}

The following inequalities are helpful to get good estimates on functions $u \in \mathcal{E}_{n}$ and on the length of the intervals $I_{i}$. We first obtain for any function $u \in H^{2}(a, b)$, a lower estimate on the $L^{2}$-norm of $u^{\prime \prime}$ by comparing $u$ with a third degree polynomial which coincides (in $H^{2}(a, b)$ ) with $u$ at both extremities.

Lemma 4. Given an interval $[a, b] \subset \mathbb{R}$ and a function $u \in H^{2}(a, b)$ such that $u(a)=A, u(b)=B, u^{\prime}(a)=$ $A_{1}, u^{\prime}(b)=B_{1}$, the following inequality holds

$$
\int_{a}^{b} u^{\prime \prime 2} \mathrm{~d} x \geqslant \frac{4}{b-a}\left[\left(B_{1}-A_{1}\right)^{2}+3\left(\frac{B-A}{b-a}-A_{1}\right)\left(\frac{B-A}{b-a}-B_{1}\right)\right],
$$

and equality holds if and only if $u$ is a third degree polynomial.

Proof. Denote by $P$ the third degree polynomial that coincides in $H^{2}$ with $u$ at points $a$ and $b$. Writing $u=P+w$, we compute

$$
\int_{a}^{b} u^{\prime \prime 2} \mathrm{~d} x=\int_{a}^{b} P^{\prime \prime 2} \mathrm{~d} x+\int_{a}^{b} w^{\prime \prime 2} \mathrm{~d} x+2 \int_{a}^{b} P^{\prime \prime} w^{\prime \prime} \mathrm{d} x
$$

Integrating $P^{\prime \prime} w^{\prime \prime}$ by parts and using the fact that $w(a)=w(b)=w^{\prime}(a)=w^{\prime}(b)=0$, we see that the last integral in (15) is actually zero. We thus obtain the inequality

$$
\int_{a}^{b} u^{\prime \prime 2} \mathrm{~d} x \geqslant \int_{a}^{b} P^{\prime \prime 2} \mathrm{~d} x
$$

and the conclusion now follows by computing the integral of $P^{\prime \prime 2}$.

The following inequality is essential to obtain a lower bound on $\mathcal{F}$ and a priori estimates for functions in $\mathcal{E}_{n}$.

Lemma 5. Let $k>0$ and $\beta \in[0, \sqrt{8 k})$. Then there exists $\varepsilon>0$ such that for any $u \in H^{2}(a, b)$, we have

$$
\int_{a}^{b}\left[\frac{1}{2}\left(u^{\prime \prime 2}-\beta u^{\prime 2}\right)+k u^{2}\right] \mathrm{d} x \geqslant \varepsilon\|u\|_{H^{2}(a, b)}^{2}-\left(\varepsilon+\frac{\beta}{2}\right)\left[u u^{\prime}\right]_{a}^{b} .
$$

Proof. Notice that for any constant $\alpha$,

$$
\int_{a}^{b}\left(u^{\prime \prime}+\alpha u\right)^{2} \mathrm{~d} x=\int_{a}^{b}\left[u^{\prime \prime 2}-2 \alpha u^{\prime 2}+\alpha^{2} u^{2}\right] \mathrm{d} x+2 \alpha\left[u^{\prime} u\right]_{a}^{b} .
$$

We then compute 


$$
\begin{aligned}
& \int_{a}^{b}\left[\frac{1}{2}\left(u^{\prime \prime 2}-\beta u^{\prime 2}\right)+k u^{2}\right] \mathrm{d} x=\varepsilon \int_{a}^{b}\left[u^{\prime \prime 2}+u^{\prime 2}+u^{2}\right] \mathrm{d} x \\
& \quad+\frac{1-2 \varepsilon}{2} \int_{a}^{b}\left[u^{\prime \prime 2}-\frac{\beta+2 \varepsilon}{1-2 \varepsilon} u^{\prime 2}+\frac{1}{4}\left(\frac{\beta+2 \varepsilon}{1-2 \varepsilon}\right)^{2} u^{2}\right] \mathrm{d} x+\left[k-\varepsilon-\frac{(\beta+2 \varepsilon)^{2}}{8(1-2 \varepsilon)}\right] \int_{a}^{b} u^{2} \mathrm{~d} x .
\end{aligned}
$$

Now, choosing $\varepsilon$ small enough, we have $k-\varepsilon-\frac{(\beta+2 \varepsilon)^{2}}{8(1-2 \varepsilon)} \geqslant 0$. Finally, using (16) with $2 \alpha=\frac{\beta+2 \varepsilon}{1-2 \varepsilon}$, we get the desired estimate.

Next we recall the continuous imbedding of $H^{1}(a, b)$ in $C(a, b)$. The dependence on the length of the interval $(a, b)$ is important in our application.

Lemma 6. Let $-\infty \leqslant a<b \leqslant+\infty$. There exists a positive constant $C$ such that for all $u \in H^{1}(a, b)$,

$$
\sup _{x \in(a, b)}|u(x)| \leqslant C\left(1+\frac{1}{b-a}\right)\|u\|_{H^{1}(a, b)} .
$$

Proof. The proof follows from Theorems VIII.7, VIII.5 of [4].

\subsection{Behaviour of local minimizers close to the equilibria}

We now describe the oscillatory nature of the local minimizers close to a saddle focus equilibrium. To fix the ideas and to simplify the notation, we assume that $f$ is a potential for which 0 is a nondegenerate global minimum and $g$ is such that 0 is a saddle focus equilibrium of the linear equation

$$
u^{\prime \prime \prime \prime}-g(0) u^{\prime \prime}+f^{\prime \prime}(0) u=0,
$$

i.e. $g(0)^{2}<4 f^{\prime \prime}(0)$. Basically, the following lemma shows that the minimizers of the functional

$$
\mathcal{I}_{a, b}(u)=\int_{a}^{b}\left[\frac{1}{2}\left[\left(u^{\prime \prime 2}\right)+g(u) u^{\prime 2}\right]+f(u)\right] \mathrm{d} x
$$

on the set $\mathcal{E}_{a, b}=\left\{v \in H^{2}(a, b) \mid\left(u(a), u^{\prime}(a)\right)=y_{0}\right.$ and $\left.\left(u(b), u^{\prime}(b)\right)=y_{1}\right\}$ are small (for the $C^{3}$-norm) whenever $y_{0}$ and $y_{1}$ are small. It then follows that the oscillatory behaviour of the solutions of the linearization (18) around the equilibrium extends to these minimizers. The following lemma is adapted from Theorem 4.1 [8] where it is assumed that $g$ is positive.

Lemma 7. Let $f$ and $g \in C^{2}(\mathbb{R})$ be such that $f(0)=f^{\prime}(0)=0$ and assume that for some $k>0, \beta \in[0, \sqrt{8 k})$ and all $u \in \mathbb{R}$,

$$
f(u) \geqslant k u^{2} \text { and } g(u) \geqslant-\beta .
$$

Then, there exist $\delta_{0}>0$ and $S>1$ such that if $b-a \geqslant 1,\left\|y_{0}\right\| \leqslant \delta_{0},\left\|y_{1}\right\| \leqslant \delta_{0}$ and $u$ minimizes $\mathcal{I}_{a, b}$ on $\mathcal{E}_{a, b}$, we have

$$
\|u\|_{C^{3}([a, b])} \leqslant S \max \left(\left\|y_{0}\right\|,\left\|y_{1}\right\|\right) .
$$

Moreover, if $g(0)^{2}<4 f^{\prime \prime}(0)$, there exists $\tau_{0}>0$ such that if $b-a \geqslant 1$ and $\max \left(\left\|y_{0}\right\|,\left\|y_{1}\right\|\right)>0$, u changes sign on every subinterval of $[a, b]$ having length larger than $\tau_{0}$. 
Proof. Throughout the proof, $C$ is a positive constant that may change from line to line. Let us first prove the estimate (19). As 0 is a nondegenerate minimum of $f$, there exist $\delta_{1}>0$ and $\eta>0$ such that $\left|f^{\prime}(u)\right| \leqslant 2 \eta|u|$ and $f(u) \leqslant \eta u^{2}$ for $|u| \leqslant \delta_{1}$.

Claim 1. There exists $C_{1}>0$ and $\delta_{2}>0$ such that if $\left\|y_{0}\right\| \leqslant \delta \leqslant \delta_{2}$ and $\left\|y_{1}\right\| \leqslant \delta \leqslant \delta_{2}$, then $\inf _{\mathcal{E}_{a, b}} \mathcal{I}_{a, b} \leqslant C_{1} \delta^{2}$.

Define $P(x)$ as follows

$$
P(x)= \begin{cases}P_{0}(x) & \text { if } a \leqslant x \leqslant a+\frac{1}{2}, \\ 0 & \text { if } a+\frac{1}{2}<x \leqslant b-\frac{1}{2}, \\ P_{1}(x) & \text { if } b-\frac{1}{2}<x \leqslant b,\end{cases}
$$

where $P_{i}, i=0,1$, are the third degree polynomia satisfying $\left(P_{0}(a), P_{0}^{\prime}(a)\right)=y_{0},\left(P_{1}(b), P_{1}^{\prime}(b)\right)=y_{1}$ and $\left(P_{0}\left(a+\frac{1}{2}\right), P_{0}^{\prime}\left(a+\frac{1}{2}\right)\right)=\left(P_{1}\left(b-\frac{1}{2}\right), P_{1}^{\prime}\left(b-\frac{1}{2}\right)\right)=(0,0)$. Observe that there exists $0<\delta_{2} \leqslant \delta_{1}$ such that if $\left\|y_{0}\right\| \leqslant \delta \leqslant \delta_{2}$ and $\left\|y_{1}\right\| \leqslant \delta \leqslant \delta_{2}$, then $\|P\|_{L^{\infty}} \leqslant \delta_{1}$. It then follows from an easy computation that

$$
\inf _{\mathcal{E}_{a, b}} \mathcal{I}_{a, b} \leqslant \mathcal{I}_{a, b}(P) \leqslant C_{1} \delta^{2},
$$

where $C_{1}>0$ essentially depends on $\eta$ and $\|g\|_{L^{\infty}\left(-\delta_{1}, \delta_{1}\right)}$.

Claim 2. There exists $C_{2}>0$ and $\delta_{0}>0$ such that if $u$ is a minimizer in $\mathcal{E}_{a, b}$ with $\left\|y_{0}\right\| \leqslant \delta \leqslant \delta_{0}$ and $\left\|y_{1}\right\| \leqslant \delta \leqslant \delta_{0}$, then $\|u\|_{C^{3}} \leqslant C_{2} \delta$.

First, notice that the inequality of Lemma 5 implies that $\mathcal{I}_{a, b}$ is bounded from below on $\mathcal{E}_{a, b}$. Therefore, the existence of a minimizer follows by standard arguments. Moreover, if $u$ is a minimizer, $u$ solves $(9)$ on $[a, b]$ and satisfies the boundary conditions $\left(u(a), u^{\prime}(a)\right)=y_{0}$ and $\left(u(b), u^{\prime}(b)\right)=y_{1}$. Assuming that $\left\|y_{0}\right\| \leqslant \delta \leqslant \delta_{0}$ and $\left\|y_{1}\right\| \leqslant \delta \leqslant \delta_{0}$, we now deduce by using the inequality of Lemma 5 that

$$
\|u\|_{H^{2}} \leqslant C \delta
$$

and from Lemma 6, we obtain

$$
\|u\|_{C^{1}} \leqslant C \delta .
$$

Observe that we can choose a constant $C$ that does not depend on the length of $[a, b]$ as $b-a \geqslant 1$. Taking $\delta_{0} \leqslant \delta_{1} / C$, we infer that $\|u\|_{\infty} \leqslant \delta_{1}$ and therefore the differential equation (9) yields the estimate

$$
\left\|u^{\prime \prime \prime \prime \prime}\right\|_{L^{2}} \leqslant C \delta
$$

with $C$ independent of $[a, b]$. We now conclude by interpolation that

$$
\left\|u^{\prime \prime \prime}\right\|_{L^{2}} \leqslant C\left(\left\|u^{\prime \prime \prime \prime}\right\|_{L^{2}}+\left\|u^{\prime \prime}\right\|_{L^{2}}\right) \leqslant C \delta .
$$

The constant in the interpolation inequality can be taken independently on $[a, b]$ as $b-a \geqslant 1$, see [1], so that $C$ does not depend on $[a, b]$. Now, the bound in $C^{3}$ follows from the bound in $H^{4}$ and Lemma 6 .

The last statement of the theorem is included in Theorem 4.2 in [8]. An alternative proof can be found in [3].

We also need the equivalent of Lemma 7 with the settings of Theorem 2.

Lemma 8. Let $f$ and $g \in C^{2}(\mathbb{R})$ be such that $f(u) \geqslant 0$ for all $u \in \mathbb{R}, f(0)=f^{\prime}(0)=0$ and assume that for some function $\tilde{g} \in C(\mathbb{R})$ and some $k<1$,

$$
g(u) \geqslant \tilde{g}(u), \quad|\widetilde{G}(u)| \leqslant k \sqrt{8 f(u)}, \quad \forall u \in \mathbb{R},
$$


where $\widetilde{G}(u):=\int_{0}^{u} \tilde{g}(s) \mathrm{d}$ s. Assume moreover that $g(0)^{2}<4 f^{\prime \prime}(0)$. Then the conclusion of Lemma 7 holds.

Proof. The proof is similar to that of Lemma 7. Though the potential is not bounded from below by a parabola everywhere, we have good estimates close to 0 . To prove Claim 2, a computation similar to those of Lemma 4 in [2] shows that for $u \in \mathcal{E}_{a, b}$,

$$
\mathcal{I}_{a, b}(u) \geqslant \frac{1}{2}\left[\widetilde{G}(u(b)) u^{\prime}(b)-\widetilde{G}(u(a)) u^{\prime}(a)\right]+s \int_{a}^{b}\left[\frac{u^{\prime \prime 2}}{2}+f(u)\right] \mathrm{d} x
$$

for some $s>0$. Using this last inequality, the conclusion follows arguing as in the proof of Lemma 7.

\subsection{Clipping}

Next, we recall the clipping procedure introduced in [8]. When minimizing a functional in a certain space, we often want to be able to modify locally any function by another one which is in the same space, has better properties and lower action. When dealing with a second order equation and its associated functional, we usually only have to worry about keeping functions continuous. As our functional $\mathcal{F}$ is defined in a translate of $H^{2}\left(\mathbb{R}^{+}\right)$, things are more complicated. For example, any modification has to keep the functions at least $C^{1}$. The following lemma can be seen as a tool for performing authorized modification. We refer the reader to [8] for the proof.

Lemma 9. Let $a \leqslant s_{1}<s_{2} \leqslant s_{3}<s_{4} \leqslant b$ and let $u \in H^{2}(a, b)$ be such that $u(x) \subset[u(a), u(b)]$ for all $x \in[a, b]$, $u$ is invertible on $\left[s_{1}, s_{2}\right]$ and $\left[s_{3}, s_{4}\right]$,

$$
u\left(s_{1}\right)=u\left(s_{3}\right), \quad u\left(s_{2}\right)=u\left(s_{4}\right), \quad\left(u^{\prime}\left(s_{1}\right)-u^{\prime}\left(s_{3}\right)\right)\left(u^{\prime}\left(s_{2}\right)-u^{\prime}\left(s_{4}\right)\right) \leqslant 0 .
$$

Then there exist $s_{1} \leqslant a_{1} \leqslant s_{2} \leqslant s_{3} \leqslant b_{1} \leqslant s_{4}$ such that the function $\hat{u}:\left[a, b-\left(b_{1}-a_{1}\right)\right] \rightarrow \mathbb{R}$ defined by

$$
\hat{u}(x)= \begin{cases}u(x) & \text { if } x \in\left[a, a_{1}\right], \\ u\left(x+b_{1}-a_{1}\right) & \text { if } x \in\left(a_{1}, b-\left(b_{1}-a_{1}\right)\right]\end{cases}
$$

belongs to $H^{2}\left(a, b-\left(b_{1}-a_{1}\right)\right)$.

When $g$ is nonnegative, it is clear that the clipping procedure decreases the action. Indeed, the value of $\mathcal{F}$ on each discarded piece is positive. When $g$ takes negative values as well, this is no longer true. However, we show in the next lemma that under some assumptions, this still reduces the action when the function is of one sign on the discarded pieces.

Lemma 10. Let $f$ and $g \in C(\mathbb{R})$ be even functions such that for some $k>0, \beta \in[0, \sqrt{8 k})$ and all $u \geqslant 0$,

$$
f(u) \geqslant k(u-1)^{2} \text { and } g(u) \geqslant-\beta \text {. }
$$

Let $u \in H^{2}(a, b)$ be such that $u(a)=u(b)$ and $u^{\prime}(a)=u^{\prime}(b)$. Assume moreover that $u$ is of one sign. Then

$$
\int_{a}^{b}\left[\frac{1}{2}\left(u^{\prime \prime 2}+g(u) u^{\prime 2}\right)+f(u)\right] \mathrm{d} x \geqslant 0 \text {. }
$$

Proof. Suppose that $u$ is nonnegative on $(a, b)$. Then using the inequality of Lemma 5 applied to $u-1$, we have

$$
\int_{a}^{b}\left[\frac{1}{2}\left(u^{\prime \prime 2}+g(u) u^{\prime 2}\right)+f(u)\right] \mathrm{d} x \geqslant \int_{a}^{b}\left[\frac{1}{2}\left(u^{\prime \prime 2}-\beta u^{\prime 2}\right)+k(u-1)^{2}\right] \mathrm{d} x \geqslant \varepsilon\|u-1\|_{H^{2}(a, b)}^{2} .
$$


If $u$ is nonpositive on $(a, b)$, we obtain

$$
\int_{a}^{b}\left[\frac{1}{2}\left(u^{\prime \prime 2}+g(u) u^{\prime 2}\right)+f(u)\right] \mathrm{d} x \geqslant \varepsilon\|u+1\|_{H^{2}(a, b)}^{2} .
$$

Remark 1. It is easy to prove that Lemma 10 holds also under the assumptions of Theorem 2 . The idea is contained in [2], Lemma 4.

\section{Estimates on minimizing sequences}

We recall that $u \in \mathcal{E}_{n}$ if there exist $0=x_{0}<x_{1}<\cdots<x_{n}<x_{n+1}=\infty$ such that

$$
\begin{aligned}
& u(x)(-1)^{i+n}>0 \text { for } x \in\left(x_{i}, x_{i+1}\right), \\
& \max _{\left(x_{i}, x_{i+1}\right)} u(x)(-1)^{i+n}>1 .
\end{aligned}
$$

We still denote by $I_{i}$ the intervals $\left(x_{i}, x_{i+1}\right)$ for $i=0, \ldots, n$. To avoid some confusions, we sometimes use the notation $I_{i}^{u}$ to emphasize that these intervals correspond to the transitions of $u$.

We also introduce the notation

$$
\mathcal{F}_{I_{i}}(u):=\int_{x_{i}}^{x_{i+1}}\left[\frac{1}{2}\left(u^{\prime \prime 2}+g(u) u^{\prime 2}\right)+f(u)\right] \mathrm{d} x
$$

and for a given function $h$ and a set $A \subset \mathbb{R}$, we denote by $h^{-1}(A)$ the set $\{x \in \operatorname{dom} h \mid h(x) \in A\}$.

The aim of this section is to construct a minimizing sequence $\left(u_{p}\right)_{p}$ that has the following properties:

(a) there exists $I>0$ such that for all $u_{p},\left|I_{i}^{u_{p}}\right| \leqslant I$;

(b) there exists $C>0$ such that for all $u_{p},\left\|u_{p}-1\right\|_{H^{2}} \leqslant C$.

Throughout the section, we assume that

(F1) $f$ and $g \in C(\mathbb{R})$ are even functions such that for some $k>0, \beta \in[0, \sqrt{8 k})$ and all $u \geqslant 0, f(u) \geqslant k(u-1)^{2}$ and $g(u) \geqslant-\beta$.

First, we obtain a lower bound on $\mathcal{F}_{I_{i}}(u)$ for all $i=0, \ldots, n$.

Lemma 11. Let $M>0$ and assume that (F1) holds. There exists $K>0$ such that if $u \in \mathcal{E}_{n}$ satisfies $\mathcal{F}(u)<M$, then $\mathcal{F}_{I_{i}}(u) \geqslant-K$ for all $i=0, \ldots, n$.

Proof. Let $u$ be of function of $\mathcal{E}_{n}$ such that $\mathcal{F}(u)<M$. Suppose first that $\left|I_{i}\right| \leqslant 1 / \sqrt{\beta}$ and $0 \leqslant i \leqslant n-1$. We then compute

$$
\left\|u^{\prime}\right\|_{L^{2}\left(I_{i}\right)}^{2} \leqslant\left|I_{i}\right|^{2}\left\|u^{\prime \prime}\right\|_{L^{2}\left(I_{i}\right)}^{2} \leqslant \frac{1}{\beta}\left\|u^{\prime \prime}\right\|_{L^{2}\left(I_{i}\right)}^{2} .
$$

It follows that

$$
\mathcal{F}_{I_{i}}(u) \geqslant \frac{1}{2}\left(\left\|u^{\prime \prime}\right\|_{L^{2}\left(I_{i}\right)}^{2}-\beta\left\|u^{\prime}\right\|_{L^{2}\left(I_{i}\right)}^{2}\right) \geqslant 0 .
$$


Suppose now that $\left|I_{i}\right|>1 / \sqrt{\beta}$ (which is of course the case for $I_{n}$ ) and assume to fix the ideas that $u$ is positive on $I_{i}$. The case of an interval where $u$ is negative follows by symmetry. We deduce from Lemma 5 that

$$
\begin{aligned}
\mathcal{F}_{I_{i}}(u) & \geqslant \int_{x_{i}}^{x_{i}+1}\left[\frac{1}{2}\left(u^{\prime \prime 2}-\beta u^{\prime 2}\right)+k(u-1)^{2}\right] \mathrm{d} x \\
& \geqslant \varepsilon\|u-1\|_{H^{2}\left(I_{i}\right)}^{2}-2\left(\varepsilon+\frac{\beta}{2}\right)\left\|u^{\prime}\right\|_{L^{\infty}\left(I_{i}\right)} .
\end{aligned}
$$

Indeed, we have $u\left(x_{i}\right)=u\left(x_{i+1}\right)=0$ for $i=0, \ldots, n-1$ while remember that $x_{n+1}=\infty$ and $u\left(x_{n+1}\right)=1$.

Combining the estimate (20) with the inequality (17) of Lemma 6 applied to $u^{\prime}$ with $b-a \geqslant 1 / \sqrt{\beta}$, we obtain

$$
\mathcal{F}_{I_{i}}(u) \geqslant \varepsilon\|u-1\|_{H^{2}\left(I_{i}\right)}^{2}-2 C(1+\sqrt{\beta})\left(\varepsilon+\frac{\beta}{2}\right)\|u-1\|_{H^{2}\left(I_{i}\right)}
$$

so that the conclusion easily follows.

Observe that as a straightforward consequence of the previous lemma, we obtain for every fixed $n \in \mathbb{N}$, a lower bound on $\mathcal{F}(u)$ for all $u \in \mathcal{E}_{n}$.

From Lemma 11, we now deduce a lower estimate on the length of the intervals $I_{i}$.

Lemma 12. Let $M>0$ and assume that (F1) holds. Then, there exists $\eta>0$ such that if $u \in \mathcal{E}_{n}$ satisfies $\mathcal{F}(u)<M$, then $\left|I_{i}\right| \geqslant \eta$ for all $i=0, \ldots, n$.

Proof. Let $u$ be a function of $\mathcal{E}_{n}$ such that $\mathcal{F}(u)<M$. Let

$$
\eta=\min \left(\sqrt[3]{\frac{3}{2(M+n K)},} \frac{1}{\sqrt{2 \beta}}\right),
$$

where $K$ is given by Lemma 11 and suppose by contradiction that $\left|I_{i_{0}}\right|<\eta$ for some $0 \leqslant i_{0} \leqslant n-1$. As $\eta \leqslant \frac{1}{\sqrt{2 \beta}}$, we infer that $\left\|u^{\prime}\right\|_{L^{2}\left(I_{i_{0}}\right)}^{2} \leqslant \frac{1}{2 \beta}\left\|u^{\prime \prime}\right\|_{L^{2}\left(I_{i_{0}}\right)}^{2}$ and

$$
\mathcal{F}_{I_{i_{0}}}(u) \geqslant \frac{1}{4}\left\|u^{\prime \prime}\right\|_{L^{2}\left(I_{i_{0}}\right)}^{2}
$$

We now estimate the $L^{2}$-norm of $u^{\prime \prime}$ by help of Lemma 4. To fix the ideas, we suppose again that $u$ is positive on $I_{i_{0}}$. By definition of $\mathcal{E}_{n}$, we know that for some $\bar{x}_{i_{0}} \in I_{i_{0}}, u\left(\bar{x}_{i_{0}}\right)=\max _{I_{i_{0}}} u>1$. Taking $A=u\left(x_{i_{0}}\right)=0, B=$ $u\left(\bar{x}_{i_{0}}\right), A_{1}=u^{\prime}\left(x_{i_{0}}\right), B_{1}=u^{\prime}\left(\bar{x}_{i_{0}}\right)=0$ and $\tau_{i_{0}}=\bar{x}_{i_{0}}-x_{i_{0}}$, we deduce from Lemma 4 that

$$
\int_{x_{i_{0}}}^{\bar{x}_{i_{0}}} u^{\prime \prime 2} \mathrm{~d} x \geqslant \frac{4}{\tau_{i_{0}}}\left(\left(u^{\prime}\left(x_{i_{0}}\right)\right)^{2}+3\left(\frac{u\left(\bar{x}_{i_{0}}\right)}{\tau_{i_{0}}}-u^{\prime}\left(x_{i_{0}}\right)\right) \frac{u\left(\bar{x}_{i_{0}}\right)}{\tau_{i_{0}}}\right) \geqslant \frac{3 u^{2}\left(\bar{x}_{i_{0}}\right)}{\tau_{i_{0}}^{3}} .
$$

A similar inequality holds for the integral between $\bar{x}_{i_{0}}$ and $x_{i_{0}+1}$ so that, taking (22) into account, we obtain

$$
\mathcal{F}_{I_{i_{0}}}(u) \geqslant \frac{3 u^{2}\left(\bar{x}_{i_{0}}\right)}{2\left|I_{i_{0}}\right|^{3}}
$$

Now, as $\mathcal{F}_{I_{i}}(u) \geqslant-K$ for all other $i$ 's, we get a contradiction as

$$
\mathcal{F}(u) \geqslant \frac{3}{2 \eta^{3}}-n K \geqslant M
$$


The previous lower estimate on the length of the intervals $I_{i}$ implies, for every function $u \in \mathcal{E}_{n}$ that satisfies $\mathcal{F}(u)<M$, a uniform upper bound on $\|u-1\|_{H^{2}\left(I_{i}\right)}$ or $\|u+1\|_{H^{2}\left(I_{i}\right)}$.

Lemma 13. Let $M>0$ and (F1) hold. There exists $N>0$ such that if $u \in \mathcal{E}_{n}$ satisfies $\mathcal{F}(u)<M$, then for all $i=0, \ldots, n$,

$$
\|u-1\|_{H^{2}\left(I_{i}\right)} \leqslant N
$$

on every interval $I_{i}$ where $u$ is positive, while

$$
\|u+1\|_{H^{2}\left(I_{i}\right)} \leqslant N
$$

for those $I_{i}$ where $u$ is negative.

Proof. Let $u \in \mathcal{E}_{n}$ be such that $\mathcal{F}(u)<M$. We first deduce from Lemma 12 the existence of $\eta>0$ such that $\left|I_{i}\right| \geqslant \eta$ for all $i=0, \ldots, n$. We can therefore obtain an estimate similar to (21) when $u$ is positive on $I_{i}$. An estimate concerning the $H^{2}$-norm of $u+1$ holds on intervals $I_{i}$ where $u$ is negative.

From Lemma 11 , we also know that for all $i=0, \ldots, n, \mathcal{F}_{I_{i}}(u) \geqslant-K$ for some positive constant $K$ independent of $i$. It follows that for each $i=0, \ldots, n$, we have

$$
\mathcal{F}_{I_{i}}(u) \leqslant \mathcal{F}(u)+n K
$$

and therefore, if $u$ is positive on $I_{i}$, we have for some constant $C>0$

$$
\varepsilon\|u-1\|_{H^{2}\left(I_{i}\right)}^{2}-C\|u-1\|_{H^{2}\left(I_{i}\right)} \leqslant M+n K
$$

and similarly

$$
\varepsilon\|u+1\|_{H^{2}\left(I_{i}\right)}^{2}-C\|u+1\|_{H^{2}\left(I_{i}\right)} \leqslant M+n K
$$

on the intervals $I_{i}$ where $u$ is negative. These two estimates imply the desired a priori bounds.

The success of the minimization process depends on a control on the length of the intervals $I_{i}$ to prevent from a loss of compactness. In fact, even for a function $u \in \mathcal{E}_{n}$ that satisfies $\mathcal{F}(u)<M$, we cannot obtain a bound on the length of the intervals $I_{i}^{u}$ because if $u$ is very close to \pm 1 with small derivatives on a long interval, the action along this interval can be arbitrarily small. However, we obtain a control on the length of the transitions of any function of a minimizing sequence by locally modifying it where it is too close to one of the equilibria. The key arguments for such modifications are the oscillatory nature of the minimizers close to the equilibria and the clipping procedure.

Lemma 14. Let $f$ and $g \in C^{2}(\mathbb{R})$ be even functions such that $f(1)=0, g^{2}(1)<4 f^{\prime \prime}(1)$ and assume that (F1) holds. Let $M>0$ and $u \in \mathcal{E}_{n}$ be such that $\mathcal{F}(u)<M$. Then there exists $I>0$ and $v \in \mathcal{E}_{n}$ such that for all $i=0, \ldots, n-1,\left|I_{i}^{v}\right| \leqslant I$ and $\mathcal{F}(v) \leqslant \mathcal{F}(u)$.

Proof. Let $\delta_{0}>0, \tau_{0}>0$ and $S>1$ be given by Lemma 7 and take $\varepsilon>0$ such that $\varepsilon \leqslant \delta_{0} / S$. Let $u \in \mathcal{E}_{n}$ be a function that satisfies $\mathcal{F}(u)<M$. To keep the ideas as clear as possible, we only focus on one interval $I_{i}^{u}$ for some $0 \leqslant i \leqslant n-1$ and we assume that $u$ is positive on $I_{i}^{u}$.

Step 1 . We replace $u$ by a function $w$ whose ith bump takes at most two times each of the values $1-\varepsilon$ and $1+\varepsilon$. By definition of the class $\mathcal{E}_{n}$, there exists $\bar{x}_{i} \in I_{i}$ such that $\max _{I_{i}} u=u\left(\bar{x}_{i}\right)>1$. We first locally replace $u$ at the left of $\bar{x}_{i}$ by a function $\breve{u} \in \mathcal{E}_{n}$ such that $\check{u}_{\left.\right|_{\left(x_{i}, \bar{x}_{i}\right)} ^{-1}}([1-\varepsilon, 1+\varepsilon])$ is an interval and $\mathcal{F}(\breve{u}) \leqslant \mathcal{F}(u)$. The idea is to use the clipping procedure to discard the possible oscillations of $u_{\left.\right|_{\left(x_{i}, \bar{x}_{i}\right)}}$ around $1-\varepsilon$ and $1+\varepsilon$. As we modify a positive function, we infer from Lemma 10 that the clipping process decreases the action. Suppose that $u$ crosses $1-\varepsilon$ more that one time. We then define 


$$
\begin{aligned}
& \xi_{2}=\min \left\{x \in\left[x_{i}, \bar{x}_{i}\right) \mid u^{\prime}(x)=0 \text { and } u(x) \geqslant 1-\varepsilon\right\}, \\
& \xi_{4}=\max \left\{x \in\left[\xi_{2}, \bar{x}_{i}\right] \mid u(x)=u\left(\xi_{2}\right)\right\}, \\
& \xi_{3}=\max \left\{x \in\left[\xi_{2}, \xi_{4}\right] \mid u^{\prime}(x)=0\right\}
\end{aligned}
$$

and

$$
\xi_{1}=\max \left\{x \in\left[x_{i}, \xi_{2}\right] \mid u(x)=u\left(\xi_{3}\right)\right\} .
$$

It is obvious that $u$ is invertible on $\left[\xi_{3}, \xi_{4}\right]$ but it could happen that $u$ has critical points in the interval [ $\left.\xi_{1}, \xi_{2}\right]$. If this is the case, we define

$$
\tilde{\xi}_{2}=\min \left\{x \in\left[\xi_{1}, \xi_{2}\right] \mid u^{\prime}(x)=0\right\}
$$

and denote by $\tilde{\xi}_{4}$ the point of the interval $\left[\xi_{3}, \xi_{4}\right]$ where $u$ takes the value $u\left(\tilde{\xi}_{2}\right)$. As $u$ is now invertible on both intervals $\left[\xi_{1}, \tilde{\xi}_{2}\right]$ and $\left[\xi_{3}, \tilde{\xi}_{4}\right]$, we are able to apply the clipping procedure of Lemma 9 . We therefore can find $s_{1} \in\left[\xi_{1}, \tilde{\xi}_{2}\right]$ and $s_{2} \in\left[\xi_{3}, \tilde{\xi}_{4}\right]$ such that $u\left(s_{1}\right)=u\left(s_{2}\right), u^{\prime}\left(s_{1}\right)=u^{\prime}\left(s_{2}\right)$. It follows that the function $\hat{u}$ defined by

$$
\hat{u}(x)= \begin{cases}u(x) & \text { if } x \in\left[0, s_{1}\right], \\ u\left(x+s_{2}-s_{1}\right) & \text { if } x \in\left(s_{1},+\infty\right)\end{cases}
$$

satisfies $\mathcal{F}(\hat{u}) \leqslant \mathcal{F}(u)$ and since $\left[\xi_{2}, \xi_{3}\right]$ is contained in $\left[s_{1}, s_{2}\right]$, the restriction of $\hat{u}$ to $\left[x_{i}, \bar{x}_{i}-\left(s_{2}-s_{1}\right)\right]$ takes the value $1-\varepsilon$ only once at the left of $\bar{x}_{i}-\left(s_{2}-s_{1}\right)$.

Repeating the same arguments to discard the possible oscillations around $1+\varepsilon$ at the left of $\bar{x}_{i}$, we obtain the desired function $\check{u}$. Now, as the same modifications can be done at the right of $\bar{x}_{i}$ the conclusion follows.

Step 2. Control on the time that $w$ spends above $1-\varepsilon$. In this step, we modify $w$ if this time is too long. Let $\theta_{1}, \theta_{4}$ be the zeros of $w_{\left.\right|_{i} ^{w}}-(1-\varepsilon)$ respectively at the left and at the right of the global maximum of $w_{\left.\right|_{I_{i}^{w}}}$. If $\max _{I_{i}^{w}} w>1+\varepsilon$, we also define $\left[\theta_{2}, \theta_{3}\right]$ as the interval where $w$ is above $1+\varepsilon$. Next, we set

$$
a_{1}=\inf \left\{x \geqslant \theta_{1}|| u(x)-1 \mid \leqslant \varepsilon \text { and }\left|u^{\prime}(x)\right| \leqslant \varepsilon\right\}
$$

and

$$
a_{2}=\sup \left\{x \leqslant \theta_{4}|| u(x)-1 \mid \leqslant \varepsilon \text { and }\left|u^{\prime}(x)\right| \leqslant \varepsilon\right\} .
$$

Notice that $a_{1}$ and $a_{2}$ need not exist. If they exist, there are three possible cases:

(i) $\theta_{1} \leqslant a_{1} \leqslant a_{2} \leqslant \theta_{2} \leqslant \theta_{3}<\theta_{4}$;

(ii) $\theta_{1} \leqslant a_{1} \leqslant \theta_{2} \leqslant \theta_{3} \leqslant a_{2} \leqslant \theta_{4}$;

(iii) $\theta_{1}<\theta_{2} \leqslant \theta_{3} \leqslant a_{1} \leqslant a_{2} \leqslant \theta_{4}$.

Claim 1. If case (i) holds, we have $a_{1}-\theta_{1} \leqslant 2, \theta_{2}-a_{2} \leqslant 2$ and $\theta_{4}-\theta_{3} \leqslant 2$. In case (ii), we have $a_{1}-\theta_{1} \leqslant 2$ and $\theta_{4}-a_{2} \leqslant 2$ while in case (iii), we have $\theta_{2}-\theta_{1} \leqslant 2, a_{1}-\theta_{3} \leqslant 2$ and $\theta_{4}-a_{2} \leqslant 2$.

Indeed, in case (i), on each of the intervals $\left[\theta_{1}, a_{1}\right],\left[a_{2}, \theta_{2}\right]$ and $\left[\theta_{3}, \theta_{4}\right]$, the variation of $w$ is smaller than $2 \varepsilon$ and $\left|w^{\prime}\right| \geqslant \varepsilon$. This implies that these intervals have length at most 2. Similar observations can be made in the other cases.

Claim 2. There exists a positive constant $C_{1}$ such that in the case where $\theta_{2}$ and $\theta_{3}$ are defined, $\theta_{3}-\theta_{2} \leqslant C_{1}$.

Indeed, as in the previous lemmas, we have the estimate

$$
\mathcal{F}_{I_{i}}(w) \leqslant \mathcal{F}(w)+n K<M+n K,
$$


where $K>0$ is given by Lemma 11. From Lemma 13, we know that

$$
\|w-1\|_{H^{2}\left(I_{i}\right)} \leqslant N
$$

for some positive $N$ independent of $I_{i}$. We therefore deduce an a priori bound for the $L^{2}$-norm of $w^{\prime}$ on $I_{i}$, leading to the estimate

$$
\mathcal{F}_{I_{i}}(w) \geqslant-L+k \int_{I_{i}}(w-1)^{2} \mathrm{~d} x
$$

for some positive constant $L$. Now the bound for $\theta_{3}-\theta_{2}$ follows from the inequality

$$
M+L+n K \geqslant k \int_{\theta_{2}}^{\theta_{3}}(w-1)^{2} \mathrm{~d} x \geqslant k\left(\theta_{3}-\theta_{2}\right) \varepsilon^{2} .
$$

Claim 3. If $a_{2}-a_{1} \geqslant \max \left(1,8 \tau_{0}\right)$, the function $w$ can be replaced by another function $\hat{w} \in \mathcal{E}_{n}$ so that the interval $\left[\theta_{1}, \theta_{4}\right]$ is clipped out to an interval of length smaller than $6+C_{1}+\max \left(1,8 \tau_{0}\right)$. Furthermore, we have $\mathcal{F}(\hat{w}) \leqslant \mathcal{F}(w)$.

First, we observe that there exists a function $\bar{w}$ which minimizes $\mathcal{F}$ in

$$
\mathcal{E}_{1,2}^{+}=\left\{u \in \mathcal{E} \mid u=w \text { on } \mathbb{R} \backslash\left[a_{1}, a_{2}\right] \text { and } u>0 \text { on }\left[a_{1}, a_{2}\right]\right\} .
$$

In $\mathcal{E}_{1,2}^{+}$, we consider functions that are positive between $\left[a_{1}, a_{2}\right]$. The reason is that we must conserve the right number of zeros to keep the modified function in $\mathcal{E}_{n}$. Notice that as $f(u) \geqslant k(u-1)^{2}$ for $u \geqslant 0$, the analysis of Lemma 7 can be applied around the equilibrium +1 . Since $w$ and $w^{\prime}$ are sufficiently small at the points $a_{1}$ and $a_{2}$, it is easily seen that a minimizer in $\mathcal{E}_{1,2}^{+}$exists. Moreover, it satisfies the differential equation (9) on [a $\left.a_{1}, a_{2}\right]$ together with the boundary conditions

$$
\bar{w}\left(a_{1}\right)=w\left(a_{1}\right), \quad \bar{w}^{\prime}\left(a_{1}\right)=w^{\prime}\left(a_{1}\right), \quad \bar{w}\left(a_{2}\right)=w\left(a_{2}\right), \quad \bar{w}^{\prime}\left(a_{2}\right)=w^{\prime}\left(a_{2}\right)
$$

and we infer from Lemma 7 that

$$
\|\bar{w}-1\|_{C^{3}\left(\left[a_{1}, a_{2}\right]\right)} \leqslant S \varepsilon \leqslant \delta_{0} .
$$

Define

$$
a_{1}^{\prime}=\max \left\{x \leqslant a_{1}|| \bar{w}(x)-1 \mid=\delta_{0}\right\}
$$

and

$$
a_{2}^{\prime}=\min \left\{x \geqslant a_{2}|| \bar{w}(x)-1 \mid=\delta_{0}\right\} .
$$

Taking (23) into account, we then have $|\bar{w}(x)-1| \leqslant \delta_{0}$ for all $x \in\left[a_{1}^{\prime}, a_{2}^{\prime}\right]$. The definitions of $a_{1}^{\prime}$ and $a_{2}^{\prime}$ imply that $\bar{w}\left(a_{1}^{\prime}\right)=1-\delta_{0}$ and $\bar{w}\left(a_{2}^{\prime}\right)=1+\delta_{0}$ or $\bar{w}\left(a_{1}^{\prime}\right)=1+\delta_{0}$ and $\bar{w}\left(a_{2}^{\prime}\right)=1-\delta_{0}$ or $\bar{w}\left(a_{1}^{\prime}\right)=1-\delta_{0}$ and $\bar{w}\left(a_{2}^{\prime}\right)=1-\delta_{0}$ (of course, we cannot have $\left.\bar{w}\left(a_{1}^{\prime}\right)=\bar{w}\left(a_{2}^{\prime}\right)=1+\delta_{0}\right)$.

In the case where $\bar{w}\left(a_{1}^{\prime}\right)=1-\delta_{0}$ and $\bar{w}\left(a_{2}^{\prime}\right)=1+\delta_{0}$, following the ideas of Step 1 , we define

$$
\begin{aligned}
& \xi_{2}=\min \left\{x \in\left[a_{1}^{\prime}, a_{2}^{\prime}\right] \mid \bar{w}^{\prime}(x)=0 \text { and } \bar{w}(x) \geqslant 1\right\}, \\
& \xi_{4}=\max \left\{x \in\left[a_{1}^{\prime}, a_{2}^{\prime}\right] \mid \bar{w}(x)=\bar{w}\left(\xi_{2}\right)\right\}, \\
& \xi_{3}=\max \left\{x \in\left[a_{1}^{\prime}, a_{2}^{\prime}\right] \mid \bar{w}^{\prime}(x)=0\right\}
\end{aligned}
$$

and take

$$
\xi_{1}=\max \left\{x \in\left[a_{1}^{\prime}, \xi_{2}\right] \mid \bar{w}(x)=\bar{w}\left(\xi_{3}\right)\right\} .
$$


Observe that Lemma 7 ensures the existence of $\xi_{2}$ and moreover $\xi_{2} \in\left[a_{1}^{\prime}, a_{1}+2 \tau_{0}\right]$. Also, $\xi_{3} \in\left[a_{2}-2 \tau_{0}, a_{2}^{\prime}\right]$. We are now able to apply the clipping procedure, defining, if needed, $\tilde{\xi}_{2}$ and $\tilde{\xi}_{4}$ in the same way as in step 1 . Consequently, we can discard the restriction of $\bar{w}$ to some interval $\left[s_{2}, s_{3}\right]$ containing $\left[\xi_{2}, \xi_{3}\right]$ and join the two remaining pieces to define a new function $v \in \mathcal{E}$ by

$$
v(x)= \begin{cases}\bar{w}(x) & \text { if } x \in\left[0, s_{2}\right], \\ \bar{w}\left(x+s_{3}-s_{2}\right) & \text { if } x \in\left(s_{2},+\infty\right) .\end{cases}
$$

Letting $\theta_{4}^{*}=\theta_{4}-\left(s_{3}-s_{2}\right)$, we have

$$
\theta_{4}^{*}-\theta_{1} \leqslant\left(a_{1}-\theta_{1}\right)+4 \tau_{0}+\left(\theta_{4}-a_{2}\right) .
$$

Observe that as $w\left(a_{2}^{\prime}\right)=\bar{w}\left(a_{2}^{\prime}\right)=1+\delta_{0}$, we had before clipping, $\max _{I_{i}} w>1+\varepsilon$ and $a_{2} \leqslant \theta_{2}$. This implies, using Claim 1, that

$$
\theta_{4}-a_{2}=\left(\theta_{4}-\theta_{3}\right)+\left(\theta_{3}-\theta_{2}\right)+\left(\theta_{2}-a_{2}\right) \leqslant 4+\left(\theta_{3}-\theta_{2}\right)
$$

so that we deduce from Claim 2 that

$$
\theta_{4}^{*}-\theta_{1} \leqslant 6+4 \tau_{0}+C_{1} .
$$

The second case is treated in the same way.

If $\bar{w}\left(a_{1}^{\prime}\right)=\bar{w}\left(a_{2}^{\prime}\right)=1-\delta_{0}$, we proceed in two steps. Let $q \in\left[a_{1}^{\prime}, a_{2}^{\prime}\right]$ be such that $\max _{x \in\left[a_{1}^{\prime}, a_{2}^{\prime}\right]} \bar{w}(x)=\bar{w}(q)$. Using Lemma 7, we know that $\bar{w}(q)>1$ and we can apply the arguments used in the first case to each of the intervals $\left[a_{1}^{\prime}, q\right]$ and $\left[q, a_{2}^{\prime}\right]$. Here, denoting by $\theta_{4}^{* *}$ the point into which $\theta_{4}$ is transformed, we have after clipping,

$$
\theta_{4}^{* *}-\theta_{1} \leqslant\left(a_{1}-\theta_{1}\right)+8 \tau_{0}+\left(\theta_{4}-a_{2}\right)
$$

and since we obviously had $a_{1} \leqslant \theta_{2} \leqslant \theta_{3} \leqslant a_{2}$ before the clipping, we deduce from Claim 1 that

$$
\theta_{4}^{* *}-\theta_{1} \leqslant 4+8 \tau_{0} \text {. }
$$

In any case, we have built a new function $v$ that satisfies the claim.

Conclusion. After the successive modifications of the previous steps, we obtain a function $v \in \mathcal{E}_{n}$ that has the following properties. Still denoting by $x_{j}, j=0, \ldots, n$, the zeros of $v$, we have $\left.v\right|_{\left(x_{i}, x_{i+1}\right)}{ }^{-1}([1-\varepsilon, 1+\varepsilon])=$ $\left[\omega_{i_{1}}, \omega_{i_{2}}\right] \cup\left[\omega_{i_{3}}, \omega_{i_{4}}\right]$ with possibly $\omega_{i_{2}}=\omega_{i_{3}}$ if $\max _{I_{i}^{v}} v \leqslant 1+\varepsilon$ and

$$
\omega_{i_{4}}-\omega_{i_{1}} \leqslant 6+C_{1}+\max \left(1,8 \tau_{0}\right) .
$$

Now, it follows from the arguments of Claim 2 in step 2 that $\omega_{i_{1}}-x_{i} \leqslant C_{1}$ and $x_{i+1}-\omega_{i_{4}} \leqslant C_{1}$. Hence,

$$
\left|I_{i}^{v}\right| \leqslant 6+3 C_{1}+\max \left(1,8 \tau_{0}\right),
$$

which proves that we can choose $I=6+3 C_{1}+\max \left(1,8 \tau_{0}\right)$.

As a direct consequence of the preceding lemma, we deduce that the right endpoint of the interval $I_{n-1}$ does not go to infinity along a minimizing sequence. This fact is of course essential for the construction of a minimizing sequence that satisfies (a) and (b). The following proposition summarizes in some sense all the previous lemmas.

Proposition 15. Let $f$ and $g \in C^{2}(\mathbb{R})$ be such that $f(1)=0, g^{2}(1)<4 f^{\prime \prime}(1)$ and assume that (F1) holds. Then, for each $n \in \mathbb{N}$, there exists $I>0, C>0$ and a minimizing sequence $\left(u_{p}\right)_{p} \subset \mathcal{E}_{n}$ such that for all $p \in \mathbb{N}$,

$$
\left|I_{i}^{u_{p}}\right| \leqslant I \quad \text { for all } i=0, \ldots, n-1
$$

and

$$
\left\|u_{p}-1\right\|_{H^{2}} \leqslant C
$$


Proof. Let $n \in \mathbb{N}$ be fixed. From Lemma 11, we know that

$$
c=\inf _{\mathcal{E}_{n}} \mathcal{F} \geqslant-(n+1) K
$$

for some positive constant $K$. Let $\left(u_{p}\right)_{p} \subset \mathcal{E}_{n}$ be a minimizing sequence. We can assume without loss of generality that for all $p \in \mathbb{N}$,

$$
\mathcal{F}\left(u_{p}\right)<c+1 \text {. }
$$

Therefore, all the previous lemmas, with $M=c+1$, apply to the functions $u_{p}$. It follows from Lemma 14 that we can choose the sequence $\left(u_{p}\right)_{p}$ in such a way that (24) holds.

To prove (25), we first observe that $x_{n}$, the right endpoint of $I_{n-1}$, is such that $x_{n} \leqslant n I$. On the other hand, we also know from Lemma 12 and 13 that there exist $\eta>0$ and $N>0$ such that $I_{i}^{u_{p}} \geqslant \eta$ for all $i=0, \ldots, n$ and all $p \in \mathbb{N}$, and

$$
\left\|u_{p}-1\right\|_{H^{2}\left(I_{i}^{u} u_{p}\right)} \leqslant N
$$

on any interval $I_{i}^{u_{p}}$ where $u_{p}$ is positive, while

$$
\left\|u_{p}+1\right\|_{H^{2}\left(I_{i}^{u}\right)}^{u_{p}} \leqslant N
$$

on those $I_{i}^{u_{p}}$ where $u_{p}$ is negative. Now, using the inequality (17) and the fact that $I_{i}^{u_{p}} \geqslant \eta$, we deduce the existence of a positive constant $R$ such that

$$
\left\|u_{p}\right\|_{\infty} \leqslant R \text {. }
$$

Denoting by $J^{+}$, respectively $J^{-}$, the set of indexes $i \in(0, \ldots, n)$ corresponding to intervals $I_{i}^{u_{p}}$ where $u_{p}$ is positive, respectively negative, we have

$$
\begin{aligned}
\left\|u_{p}-1\right\|_{H^{2}}^{2} & =\int_{0}^{\infty}\left[u_{p}^{\prime \prime 2}+u_{p}^{\prime 2}+\left(u_{p}-1\right)^{2}\right] \mathrm{d} x \\
& =\sum_{i \in J^{+}} \int_{I_{i}^{u p}}\left[u_{p}^{\prime \prime 2}+u_{p}^{\prime 2}+\left(u_{p}-1\right)^{2}\right] \mathrm{d} x+\sum_{i \in J^{-}} \int_{I_{i}^{u p}}\left[u_{p}^{\prime \prime 2}+u_{p}^{\prime 2}+\left(u_{p}+1\right)^{2}-4 u_{p}\right] \mathrm{d} x \\
& \leqslant(n+1) N-4 \sum_{i \in J^{-}} \int_{I_{i}^{u p}} u_{p} .
\end{aligned}
$$

Taking (24) and (26) into account, we finally get

$$
\left\|u_{p}-1\right\|_{H^{2}}^{2} \leqslant(n+1) N+4 m R I,
$$

where $m$ is the number of indexes in $J^{-}$.

\section{Existence of the local minimizers}

We have now almost all the ingredients to prove Theorem 1. To complete the minimizing process in a given class $\mathcal{E}_{n}$, we choose a minimizing sequence $\left(u_{p}\right)_{p} \subset \mathcal{E}_{n}$ that has the properties described in Proposition 15. From the a priori bound on $\left\|u_{p}-1\right\|_{H^{2}}$, we deduce that up to a subsequence, $u_{p}$ converges (in a way which is made 
precise below) to some function $u \in \mathcal{E}$. To prove that $u \in \mathcal{E}_{n}$, we require $\mathcal{F}$ to be lower bounded in $\mathcal{E}$. Observe that this last assumption was not used until now and actually, we do not know whether it is necessary or not.

Lemma 16. Assume that (F1) holds. Let $u \in H^{2}(a, b)$ be such that $u(a)=u(b)=0$. Assume moreover that $\inf _{\mathcal{E}} \mathcal{F}>-\infty$. Then

$$
\mathcal{F}_{a, b}(u)=\int_{a}^{b}\left[\frac{1}{2}\left(u^{\prime \prime 2}+g(u) u^{\prime 2}\right)+f(u)\right] \mathrm{d} x \geqslant 0 .
$$

Proof. Let $v \in \mathcal{E}$ be such that $v^{\prime}(0)=u^{\prime}(b)$. Suppose that $\mathcal{F}_{a, b}(u)=-c<0$ and let $n \in \mathbb{N}$ be such that $\mathcal{F}(v)-(2 n+1) c<\inf _{\mathcal{E}} \mathcal{F}$. Let us set $T=b-a$. Then defining $u^{*} \in \mathcal{E}$ by

$$
u^{*}(x)= \begin{cases}u(a+x) & \text { if } x \in[0, T), \\ -u(a+2 i T-x) & \text { if } x \in[(2 i-1) T, 2 i T), i=1, \ldots, n, \\ u(a+x-2 i T) & \text { if } x \in[2 i T,(2 i+1) T), i=1, \ldots, n, \\ v(x-(2 n+1) T) & \text { if } x \geqslant(2 n+1) T\end{cases}
$$

we obtain a contradiction as

$$
\mathcal{F}\left(u^{*}\right)=(2 n+1) \mathcal{F}_{a, b}(u)+\mathcal{F}(v)<\inf _{\mathcal{E}} \mathcal{F} .
$$

Proof of Theorem 1. Let $\left(u_{p}\right)_{p} \subset \mathcal{E}_{n}$ be a minimizing sequence that has the properties stated in Proposition 15 .

Step 1 . The sequence $\left(u_{p}\right)_{p}$ converges in $C_{\text {loc }}^{1}$ to some function $u \in \mathcal{E}$ that satisfies $\mathcal{F}(u) \leqslant \inf _{\mathcal{E}_{n}} \mathcal{F}$. From the a priori bound on $\left\|u_{p}-1\right\|_{H^{2}}$, we deduce that up to a subsequence,

$$
u_{p}-1 \stackrel{H^{2}}{\rightarrow} u-1 \quad \text { and } \quad u_{p} \stackrel{C_{\mathrm{loc}}^{1}}{\longrightarrow} u
$$

for some function $u \in \mathcal{E}$.

Let $J_{1}=[0, n I]$ and $J_{2}=(n I,+\infty)$ where $I$ is given in Proposition 15 . We then write

$$
\begin{aligned}
\mathcal{F}\left(u_{p}\right) & =\mathcal{F}_{J_{1}}\left(u_{p}\right)+\mathcal{F}_{J_{2}}\left(u_{p}\right) \\
& =\int_{J_{1}}\left[\frac{1}{2}\left(u_{p}^{\prime \prime 2}+g\left(u_{p}\right) u_{p}^{\prime 2}\right)+f\left(u_{p}\right)\right] \mathrm{d} x+\int_{J_{2}}\left[\frac{1}{2}\left(u_{p}^{\prime \prime 2}+g\left(u_{p}\right) u_{p}^{\prime 2}\right)+f\left(u_{p}\right)\right] \mathrm{d} x .
\end{aligned}
$$

Observe first that

$$
\mathcal{F}_{J_{1}}(u) \leqslant \liminf _{p \rightarrow \infty} \mathcal{F}_{J_{1}}\left(u_{p}\right) .
$$

On $J_{2}$, we know that $u_{p}$ is positive. We therefore write

$$
\begin{aligned}
\mathcal{F}_{J_{2}}\left(u_{p}\right)= & \frac{1}{2} \int_{J_{2}}\left(u_{p}^{\prime \prime 2}-\beta u_{p}^{\prime 2}+\frac{\beta^{2}}{4}\left(u_{p}-1\right)^{2}\right) \mathrm{d} x+\int_{J_{2}} \frac{g\left(u_{p}\right)+\beta}{2} u_{p}^{\prime 2} \mathrm{~d} x+\int_{J_{2}}\left(f\left(u_{p}\right)-\frac{\beta^{2}}{8}\left(u_{p}-1\right)^{2}\right) \mathrm{d} x \\
= & \frac{1}{2}\left[\int_{J_{2}}\left(u_{p}^{\prime \prime}+\frac{\beta}{2}\left(u_{p}-1\right)\right)^{2} \mathrm{~d} x-\beta u_{p}^{\prime}(n I) u_{p}(n I)\right] \\
& +\int_{J_{2}} \frac{g\left(u_{p}\right)+\beta}{2} u_{p}^{\prime 2} \mathrm{~d} x+\int_{J_{2}}\left(f\left(u_{p}\right)-\frac{\beta^{2}}{8}\left(u_{p}-1\right)^{2}\right) \mathrm{d} x .
\end{aligned}
$$


In the last equality, the first integral is convex and Fatou's Lemma is applicable to the last two so that taking also (27) into account, we deduce that $\mathcal{F}(u) \leqslant \inf _{\mathcal{E}_{n}} \mathcal{F}$.

We denote the extremities of the intervals $I_{i}^{u_{p}}$ by $x_{i}^{p}, i=0, \ldots, n$. It is clear by uniform convergence that up to a subsequence, for all $i=1, \ldots, n, x_{i}^{p}$ converges to some $x_{i} \in J_{1}$. Remember that by convention, we set $x_{0}^{p}=x_{0}=0$ and $x_{n+1}^{p}=x_{n+1}=\infty$. We call $I_{i}$ the intervals $\left(x_{i}, x_{i+1}\right), i=0, \ldots, n$. We also deduce from the convergence in $C^{1}\left(J_{1}\right)$ and the convergence in $C_{\text {loc }}^{1}\left(J_{2}\right)$ that

$$
\begin{aligned}
& u(x)(-1)^{i+n} \geqslant 0 \text { for } x \in\left(x_{i}, x_{i+1}\right), \\
& \max _{\left(x_{i}, x_{i+1}\right)} u(x)(-1)^{i+n} \geqslant 1 .
\end{aligned}
$$

Step 2. Elimination of the zeros of $u$ after $x_{n}$. If $u$ has zeros after $x_{n}$, we first modify it to keep only one of those zeros. So, suppose that $u$ vanishes at least two times after $x_{n}$. We then define

$$
a_{1}=\min \left\{x>x_{n} \mid u(x)=0\right\} \quad \text { and } \quad a_{2}=\max \left\{x>x_{n} \mid u(x)=0\right\} .
$$

Observe that as $u \in \mathcal{E}, a_{2}$ is well defined. Since $u\left(a_{1}\right)=u\left(a_{2}\right)=u^{\prime}\left(a_{1}\right)=u^{\prime}\left(a_{2}\right)=0$ by convergence in $C_{\text {loc }}^{1}$, the interval $\left[a_{1}, a_{2}\right]$ can be clipped out and the resulting function has only one zero after $x_{n}$. Moreover the function $u$ is nonnegative on the clipped interval so that this modification decreases the action.

Assume now that $u$ vanishes at some point $\xi>x_{n}$. We then have $u^{\prime}(\xi)=0$. Now as $u\left(x_{n}\right)=u(\xi)$, there exists at least one critical point $y$ between $x_{n}$ and $\xi$ such that $u(y)>0$. Here, we have two possibilities, either $y$ can be taken in such a way that $u(y) \leqslant 1$ or $[0,1]$ does not contain any critical value of $u_{\mid\left[x_{n}, \xi\right]}$.

Suppose first that we can find $y \in\left(x_{n}, \xi\right)$ such that $0<u(y) \leqslant 1$ and $u^{\prime}(y)=0$. As $u \in \mathcal{E}$,

$$
\lim _{x \rightarrow \infty}\left(u(x), u^{\prime}(x)\right)=(1,0) .
$$

Hence, we infer from Lemma 7 that $u(x)$ oscillates around 1 for $x$ large enough. Therefore, we can clip out an interval containing $[y, \xi]$ in such a way that the function obtained after clipping does not vanish after $x_{n}$.

In the second case, we can find $y \in\left(x_{n}, \xi\right)$ such that $u(y)>1$ and if $x \in\left(x_{n}, \xi\right)$ satisfies $u^{\prime}(x)=0$, then $u(x)>1$. We now define $v \in \mathcal{E}$ by

$$
v(x)= \begin{cases}-u\left(x+x_{1}\right) & \text { if } 0 \leqslant x \leqslant \xi-x_{1}, \\ u\left(x+x_{1}\right) & \text { if } x>\xi-x_{1} .\end{cases}
$$

Observe that since $\min _{\left[x_{n}-x_{1}, \xi-x_{1}\right]} v(x)<-1$ and $v$ is negative in $\left(x_{n}-x_{1}, \xi-x_{1}\right), v$ has the right number of transitions. Also, $v$ does not vanish after $\xi-x_{1}$. On the other hand, because $u\left(x_{0}\right)=u\left(x_{1}\right)$, we deduce from Lemma 16 that

$$
\int_{x_{0}}^{x_{1}}\left[\frac{1}{2}\left(u^{\prime \prime 2}+g(u) u^{\prime 2}\right)+f(u)\right] \mathrm{d} x \geqslant 0
$$

so that $\mathcal{F}(v) \leqslant \mathcal{F}(u)$.

Step 3. Elimination of the zeros of $v$ in the bumps. We still denote by $0=x_{0}<x_{1}<\cdots<x_{n}$, the extremities of the intervals $I_{i}^{v}$ (actually, these are the intervals $I_{i}^{u}$ which have been possibly translated in step 2). Suppose that there exists $\xi \in v^{-1}(0)$ so that $\xi \neq x_{i}$ for any $i=0, \ldots, n$. Hence, $\xi$ lies in the interior of an interval $I_{i}$. To fix the ideas, we assume that $v$ is nonnegative therein and denoting by $\bar{x}_{i}$ the maximum of $v$ over this interval we assume that $\xi$ is at the left of $\bar{x}_{i}$. Next, define $\xi_{1}=\min \left\{x \in I_{i} \mid v(x)=0\right\}$ and $\xi_{2}=\max \left\{x \in\left[\xi_{1}, \bar{x}_{i}\right] \mid v(x)=0\right\}$. It is easily seen that an interval containing $\left[\xi_{1}, \xi_{2}\right]$ can be clipped out so that the zeros can be deleted.

Step 4. Elimination of the tangencies with \pm 1 . The last condition that we have to check to be sure that $v \in \mathcal{E}_{n}$ is that $\max _{x \in I_{i}}|v(x)|>1$ for all $i=0, \ldots, n$. Assume that this condition fails to be true in one of the intervals $I_{i}$. In this interval, we thus have $\max _{x \in I_{i}}|v(x)|=1$. Let $\tau \in I_{i}$ be such that $|v(\tau)|=1$ and $v^{\prime}(\tau)=0$. To fix the ideas, 
assume that $v(\tau)=1$, the second case being treated in the same way. As the action of the function 1 is zero, we can modify $v$ without increasing its action by stretching the point $\tau$ to an interval of arbitrary length and gluing the function 1 to both extremities, see [8]. Now, we take $a_{1}$, (respectively $a_{2}$ ) at the left (respectively at the right) of $\tau$ in such a way that $0<\max _{i=1,2}\left\|\left(v\left(a_{i}\right)-1, v^{\prime}\left(a_{i}\right)\right)\right\| \leqslant \delta_{0}$ and stretch $\tau$ to an interval of length $\tau_{0}$. We still call $v$ the function obtained after gluing 1 at $\tau$ and $\tau+\tau_{0}$. It follows from Lemma 7 that the minimizers of

$$
\int_{a_{1}}^{a_{2}+\tau_{0}}\left[\frac{1}{2}\left(u^{\prime \prime 2}+g(u) u^{\prime 2}\right)+f(u)\right] \mathrm{d} x
$$

on the set of functions $u \in H^{2}\left(a_{1}, \tau_{0}+a_{2}\right)$ that satisfy $u\left(a_{1}\right)=v\left(a_{1}\right), u^{\prime}\left(a_{1}\right)=v^{\prime}\left(a_{1}\right), u\left(a_{2}+\tau_{0}\right)=v\left(a_{2}\right)$ and $u^{\prime}\left(a_{2}+\tau_{0}\right)=v^{\prime}\left(a_{2}\right)$ oscillate around 1 . If we replace $v$ locally by a minimizer, we obtain a new function $w$ such that $\max _{x \in I_{i}}|w(x)|>1$ and $\mathcal{F}(w) \leqslant \mathcal{F}(v)$.

Conclusion. It follows from the previous steps that we can construct $w \in \mathcal{E}_{n}$ such that $\mathcal{F}(w) \leqslant \mathcal{F}(u)$. Consequently, we have $\mathcal{F}(w)=\min _{\mathcal{E}_{n}} \mathcal{F}$. Now, observe that for all $h \in H^{2}\left(\mathbb{R}^{+}\right)$such that $h(0)=0$, for $t$ sufficiently small, $\mathcal{F}(w) \leqslant \mathcal{F}(w+t h)$. Indeed, assume that there exists a sequence $\left(t_{n}\right)_{n}$ tending to 0 such that $\mathcal{F}(w)>\mathcal{F}\left(w+t_{n} h\right)$. If $w$ is in the interior of the class $\mathcal{E}_{n}$, this is obviously a contradiction. In the case where $w$ is on the boundary of $\mathcal{E}_{n}$ i.e. if for some points $x_{i}, w\left(x_{i}\right)=w^{\prime}\left(x_{i}\right)=0$, then even for $t$ small, $w+t h$ can have more than one zero close to the $x_{i}$ 's so that it does not belong necessarily to $\mathcal{E}_{n}$. However for $t$ small enough, $w+t h$ has the right number of transitions and the oscillations close to the points $x_{i}$ can be erased using the clipping procedure. Therefore, for $n$ large enough, modifying $w+t_{n} h$ close to the $x_{i}$ 's if necessary, we obtain a function in $\mathcal{E}_{n}$ whose action is strictly smaller than $\mathcal{F}(w)$. This contradicts the definition of $w$.

We now deduce that $w$ is a critical point of $\mathcal{F}$. Using standard arguments, we can show that $w$ satisfies Eq. (9) on $[0, \infty)$ and it follows also from an easy computation that $w^{\prime \prime}(0)=0$. Hence, the odd extension $w^{*}$ of $w$ is a solution of (9). From the differential equation (9), we now infer that $w-1 \in H^{4}\left(\mathbb{R}^{+}\right)$so that $w^{*}$ satisfies (5) and $w^{*}$ is an heteroclinic solution of (9).

Remark 2. Using the conservation of the Hamiltonian

$$
H(u)=u^{\prime \prime \prime} u^{\prime}-\frac{1}{2} u^{\prime \prime 2}-\frac{1}{2} g(u) u^{\prime 2}+f(u)
$$

along solutions of (9), it is easily proved that each minimizer $u_{n}$ is actually in the interior of $\mathcal{E}_{n}$, i.e. each crossing with zero is transverse.

We now turn to the proof of Theorem 2 . Since many of the arguments are similar, we only sketch it.

Proof of Theorem 2. First, observe that assumption (14) implies that $\mathcal{F}(u) \geqslant 0$ for all $u \in \mathcal{E}$. This follows from the inequality

$$
\mathcal{F}(u) \geqslant s \int_{0}^{\infty}\left[\frac{u^{\prime \prime 2}}{2}+f(u)\right] \mathrm{d} x
$$

where $s>0$ which is valid for all $u \in \mathcal{E}$ and proved in [2].

Let $\left(u_{p}\right)_{p} \subset \mathcal{E}_{n}$ be a minimizing sequence for $\mathcal{F}$. The inequalities

$$
\mathcal{F}_{I_{i}}\left(u_{p}\right) \geqslant s \int_{I_{i}}\left[\frac{u_{p}^{\prime \prime 2}}{2}+f\left(u_{p}\right)\right] \mathrm{d} x
$$

then follow arguing as in Lemma 4 of [2]. Consequently, the equivalent of Lemma 11 (with $K=0$ ) and Lemma 12 hold. Next, we deduce as in Lemma 6 of [2] a priori bounds on $\left\|u_{p}\right\|_{\infty}$ and $\left\|u_{p}^{\prime}\right\|_{\infty}$. Then, using the inequality (29), 
these a priori bounds and the clipping procedure, the conclusion of Lemma 14 follows (possibly for a modification of the minimizing sequence). Therefore, the statement of Lemma 13 for $i=0, \ldots, n-1$ holds. To obtain an a priori bound in $I_{n}$, we first observe that for some $a>0, k>0$ and $\beta \in[0, \sqrt{8 k})$,

$$
f(u) \geqslant k(u-1)^{2} \text { and } g(u) \geqslant-\beta \text { for }|u-1| \leqslant a .
$$

Then arguing as in Proposition 2 of [2] and using the bound on $I_{i}$ for $i=0, \ldots, n-1$, we prove the existence of $T>0$ such that for all $u_{p}$, there exists $v_{p} \in \mathcal{E}_{n}$ that satisfies

$$
\left|v_{p}(x)-1\right| \leqslant a \text { for all } x \geqslant T
$$

and $\mathcal{F}\left(v_{p}\right) \leqslant \mathcal{F}\left(u_{p}\right)$. We then deduce an a priori bound on $\left\|v_{p}-1\right\|_{H^{2}\left(x_{n}, \infty\right)}$ using the bounds on $v_{p}$ and $v_{p}^{\prime}$ on $\left[x_{n}, T\right]$ and arguing as in the proof of Theorem 1 on $(T, \infty)$.

We thus obtain a minimizing sequence $\left(v_{p}\right)_{p} \subset \mathcal{E}_{n}$ that has the properties of Proposition 15. Now the end of the proof follows the same lines as the proof of Theorem 1. Just observe that denoting by $v$ the weak limit of $v_{p}$, the inequality $\mathcal{F}(v) \leqslant \lim _{p \rightarrow \infty} \mathcal{F}\left(v_{p}\right)$ follows by working separately on the intervals $[0, T]$ and $(T, \infty)$ using also (30).

As we mentioned in the introduction, the hypothesis $\inf _{\mathcal{E}} \mathcal{F}>-\infty$ of Theorem 1 implies that inf $\mathcal{E} \geqslant 0$, see Corollary 7 in [3]. Actually, we can even prove that $\inf _{\mathcal{E}} \mathcal{F}>0$ and consequently the multitransition kinks all have a strictly positive action. Observe also that as a straightforward consequence of a repeated use of the clipping procedure, the minimum $u_{n}$ of $\mathcal{F}$ in $\mathcal{E}_{n}$ has exactly one critical point in each interval $I_{i}$ for $i=0, \ldots, n-1$. On the other hand, in the tail, $u_{n}$ oscillates around 1 , so that there exists a sequence $\xi_{m}, m \in \mathbb{N}$, such that $u^{\prime}\left(\xi_{m}\right)=0$, $u^{\prime \prime}\left(\xi_{2 m}\right) \leqslant 0, u^{\prime \prime}\left(\xi_{2 m+1}\right) \geqslant 0, u\left(\xi_{2 m}\right)>u\left(\xi_{2 m+2}\right)$ and $u\left(\xi_{2 m+1}\right)<u\left(\xi_{2 m+3}\right)$.

Let us come back to the stationary Swift-Hohenberg equation (4). Taking the function $u(x)=\sin (\omega x)$ with $\omega^{4}=3 / 8$ and computing

$$
\int_{0}^{2 \pi / \omega}\left[\frac{1}{2}\left(u^{\prime \prime 2}+\beta u^{\prime 2}\right)+\frac{1}{4}\left(u^{2}-1\right)^{2}\right] \mathrm{d} x=\frac{(3+\sqrt{6} \beta) \pi}{6^{1 / 4} 4},
$$

it is easy to deduce that the functional $\mathcal{J}_{\beta}: \mathcal{E} \rightarrow \mathbb{R}$ defined by

$$
\mathcal{J}_{\beta}(u)=\int_{0}^{+\infty}\left[\frac{1}{2}\left[\left(u^{\prime \prime 2}\right)+\beta u^{\prime 2}\right]+\frac{1}{4}\left(u^{2}-1\right)^{2}\right] \mathrm{d} x
$$

is unbounded from below whenever $\beta<-\sqrt{3 / 2}$. In [3], it is proved that $\inf _{\mathcal{E}} \mathcal{J}_{\beta}=-\infty$ for $\beta<\beta_{0}$ where

$$
\beta_{0}=\inf \left\{\beta<0 \mid \inf _{\mathcal{E}} \mathcal{J}_{\beta} \geqslant 0\right\}
$$

For $\beta \geqslant \beta_{0}$, we obtain a family of kinks $u_{n}^{*}$ having $2 n+1$ zeros on $\mathbb{R}$.

Proposition 17. Let $\beta_{0}$ be given by (31). Then, for all $\beta \geqslant \beta_{0}$, for all $n \in \mathbb{N}, \mathcal{J}_{\beta}$ has a local minimum $u_{n} \in \mathcal{E}_{n}$ whose odd extension is a solution of (4) having exactly $2 n+1$ zeros. Moreover, $\mathcal{J}_{\beta}\left(u_{n}\right)<\mathcal{J}_{\beta}\left(u_{n+1}\right)$ for all $n \in \mathbb{N}$.

Proof. The existence of the minimizers $u_{n} \in \mathcal{E}_{n}$ follows directly from Theorem 1 and the definition of $\beta_{0}$. For all $n \in \mathbb{N}$, we denote by $u_{n}^{*}$ the odd extension of $u_{n}$ on $\mathbb{R}$. We now prove that $\mathcal{J}_{\beta}\left(u_{n}\right)<\mathcal{J}_{\beta}\left(u_{n+1}\right)$ for all $n \in \mathbb{N}$. Let $x_{1}$ be the first zero of $u_{n+1}$ in $(0, \infty)$. Then, the function $v_{n}=u_{n+1}\left(\cdot+x_{1}\right)$ belongs to $\mathcal{E}_{n}$. It follows from Lemma 16 that the action of $u_{n+1}$ on the interval $\left[x_{0}, x_{1}\right]$ is nonnegative. Therefore $\mathcal{J}_{\beta}\left(u_{n}\right) \leqslant \mathcal{J}_{\beta}\left(v_{n}\right) \leqslant \mathcal{J}_{\beta}\left(u_{n+1}\right)$. Suppose that $\mathcal{J}_{\beta}\left(u_{n}\right)=\mathcal{J}_{\beta}\left(u_{n+1}\right)$. Then $v_{n}$ is a minimizer of $\mathcal{J}_{\beta}$ in $\mathcal{E}_{n}$ and thus its odd extension $v_{n}^{*}$ is an heteroclinic solution of (4). Observe that $u_{n+1}^{*}\left(\cdot+x_{1}\right)$ also solves (4) on $\mathbb{R}$. As $u_{n+1}^{*}\left(\cdot+x_{1}\right)=v_{n}^{*}$ on $\mathbb{R}^{+}$, we obtain a contradiction with the uniqueness of the solution of the Cauchy problem. 


\section{Homoclinic connections}

Homoclinics to \pm 1 belong respectively to the functional spaces $\pm 1+H^{2}(\mathbb{R})$. We focus only on homoclinics to +1 . When minimizing the functional $\mathcal{F}$ defined by $(8)$, it is natural to search even homoclinic solutions. Indeed, suppose that $u-1 \in H^{2}(\mathbb{R})$, then there exists an even function $u^{*}$ that satisfies $u^{*}-1 \in H^{2}(\mathbb{R})$ and $\mathcal{F}\left(u^{*}\right) \leqslant \mathcal{F}(u)$. Observe that if $\bar{x}$ is a critical point of $u$, writing $J_{1}=(-\infty, \bar{x}]$ and $J_{2}=(\bar{x},+\infty)$, the action of $u$ is smaller on $J_{1}$ or on $J_{2}$. Assuming that $u$ has a lower action on $J_{1}$, we define $u^{*} \in 1+H^{2}(\mathbb{R})$ by

$$
u^{*}= \begin{cases}u(x) & \text { if } x \in J_{1}, \\ u(2 \bar{x}-x) & \text { if } x \in J_{2} .\end{cases}
$$

Now $\mathcal{F}\left(u^{*}\right)=2 \mathcal{F}_{J_{1}}(u) \leqslant \mathcal{F}_{J_{1}}(u)+\mathcal{F}_{J_{2}}(u)=\mathcal{F}(u)$.

By translation invariance, we can also restrict ourselves to even solutions with respect to zero. We therefore define the functional space

$$
\widetilde{\mathcal{E}}=\left\{u \in C^{1}\left(\mathbb{R}^{+}\right) \mid u-1 \in H^{2}\left(\mathbb{R}^{+}\right), u^{\prime}(0)=0\right\} .
$$

It is easily seen that if $u \in \widetilde{\mathcal{E}}$ is a critical point of the functional $\mathcal{F}$, then $u^{\prime \prime \prime}(0)=0$. It follows that the even extension of $u$ on $\mathbb{R}$ is a solution of (9) which is at least $C^{4}$. Also, it is not difficult to verify that the condition (6) is satisfied.

As before, we assume that $(\mathrm{F} 1)$ holds and $\mathcal{F}$ is bounded from below on $\widetilde{\mathcal{E}}$. Actually, in a saddle-foci situation, this again implies that $\mathcal{F}$ is nonnegative on $\widetilde{\mathcal{E}}$.

It is obvious that looking at minimizers of $\mathcal{F}$ in $\widetilde{\mathcal{E}}$ leads to the trivial solution $u=1$. Moreover 1 is the only function having zero action if $\mathcal{F}$ is bounded from below. To get nontrivial solutions, we minimize $\mathcal{F}$ in subclasses $\widetilde{\mathcal{E}}_{n} \subset \widetilde{\mathcal{E}}$ that do not contain the function 1 . We define for each $n \geqslant 0$ the subset $\widetilde{\mathcal{E}}_{n} \subset \widetilde{\mathcal{E}}$ consisting of functions whose even extensions on $\mathbb{R}$, make $2 n$ transitions. Precisely, we assume that $u \in \widetilde{\mathcal{E}}_{n}$ if $u \in \widetilde{\mathcal{E}}$, and there exist $0=x_{0}<x_{1}<\cdots<x_{n}<x_{n+1}=\infty$ such that

$$
\begin{aligned}
& u(x)(-1)^{i+n}>0 \text { for } x \in\left(x_{i}, x_{i+1}\right), \\
& \max _{\left(x_{i}, x_{i+1}\right)} u(x)(-1)^{i+n}>1
\end{aligned}
$$

Adapting the arguments of the previous sections, we are able to prove that $\mathcal{F}$ has a local minimum in each of these subspaces in the two following situations.

Theorem 18. Let $f$ and $g \in C^{2}(\mathbb{R})$ satisfy $(\mathrm{F} 1)$ and $g(1)^{2}<4 f^{\prime \prime}(1)$. Assume further that $\inf _{\widetilde{\mathcal{E}}} \mathcal{F}>-\infty$. Then $\mathcal{F}$ has a local minimizer $\tilde{u}_{n}$ in each subspace $\widetilde{\mathcal{E}}_{n}$. Moreover, the even extension of $\tilde{u}_{n}$ on $\mathbb{R}$ is an homoclinic solution of (9) to +1 , having exactly $2 n$ zeros.

Theorem 19. Let $f$ and $g \in C^{2}(\mathbb{R})$ be even functions such that $f(1)=f^{\prime}(1)=0$ and for some function $\tilde{g} \in C(\mathbb{R})$ and some $k<1$,

$$
g(u) \geqslant \tilde{g}(u), \quad|\widetilde{G}(u)| \leqslant k \sqrt{8 f(u)}, \quad \forall u \in \mathbb{R},
$$

where $\widetilde{G}(u):=\int_{0}^{u} \tilde{g}(s) \mathrm{d} s$. Assume moreover that $g(1)^{2}<4 f^{\prime \prime}(1)$. Then the conclusion of Theorem 18 holds.

The proofs are basically identical to those of Theorems 1 and 2. Just observe that in order to adapt the second step of Theorem 1, we need the equivalent of Lemma 16 for functions $u \in H^{2}(a, b)$ that satisfy $u^{\prime}(a)=u^{\prime}(b)=0$. Also, some arguments are slightly different, taking into account that $u^{\prime}(0)=0$ instead of $u(0)=0$ for functions $u \in \widetilde{\mathcal{E}}$. 
Observe that under the assumptions of Theorems 18 and $19, \inf _{\mathcal{E}} \mathcal{F}>-\infty$ if and only if $\inf _{\widetilde{\mathcal{E}}} \mathcal{F}>-\infty$. Therefore, we obtain homoclinic solutions for the Swift-Hohenberg equation for the same range of $\beta$ as in Proposition 17.

Proposition 20. Let $\beta_{0}$ be as in Proposition 17. Then, for all $\beta \geqslant \beta_{0}$, for all $n \in \mathbb{N}, \mathcal{J}_{\beta}$ has a local minimum $\tilde{u}_{n} \in \widetilde{\mathcal{E}}_{n}$ whose even extension is a solution of (4) homoclinic to +1 having exactly $2 n$ zeros. Moreover, $\mathcal{J}_{\beta}\left(\tilde{u}_{n}\right)<\mathcal{J}_{\beta}\left(\tilde{u}_{n+1}\right)$ for all $n \in \mathbb{N}$.

\section{References}

[1] R.A. Adams, Sobolev Spaces, Academic Press, 1975.

[2] D. Bonheure, L. Sanchez, M. Tarallo, S. Terracini, Heteroclinic connections between nonconsecutive equilibria of a fourth order differential equation, Calc. Var. Partial Differential Equations 17 (2003) 341-356.

[3] D. Bonheure, P. Habets, L. Sanchez, Minimizers for fourth order symmetric bistable equation, Atti de seminari de matematica e fisica de la universita di Modena, in press.

[4] H. Brezis, Analyse fonctionnelle, Théorie et applications, Masson, 1983.

[5] G.T. Dee, W. van Saarloos, Bistable systems with propagating fronts leading to patern formation, Phys. Rev. Lett. 60 (1988) $2641-2644$.

[6] G. Gomper, M. Schick, Phase Transitions and Critical Phenomena, Academic Press, 1994.

[7] P. Habets, L. Sanchez, M. Tarallo, S. Terracini, Heteroclinics for a class of fourth order conservative differential equations, in: CD ROM Proceedings, Equadiff 10, Prague, 2001.

[8] W.D. Kalies, J. Kwapisz, R.C.A.M. VanderVorst, Homotopy classes for stable connections between Hamiltonian saddle-focus equilibria, Comm. Math. Phys. 193 (1998) 337-371.

[9] W.D. Kalies, R.C.A.M. VanderVorst, Multitransition homoclinic and heteroclinic solutions of the extended Fisher-Kolmogorov equation, J. Differential Equations 131 (1996) 209-228.

[10] A. Kolmogorov, I. Petrovski, N. Piscounov, Étude de l'équation de la diffusion avec croissance de la quantité de matière et son application à un problème biologique, Bull. Univ. Moskow Ser. Internat. Sec. A 1 (1937) 1-25.

[11] L.A. Peletier, W.C. Troy, Spatial patterns described by the extended Fisher-Kolmogorov (EFK) equation: Kinks, Differential Integral Equations 8 (1995) 1279-1304.

[12] L.A. Peletier, W.C. Troy, A topological shooting method and the existence of kinks of the extended Fisher-Kolmogorov equation, Topological Methods Nonlin. Anal. 6 (1995) 331-355.

[13] L.A. Peletier, W.C. Troy, Spatial Patterns, Higher Order Models in Physics and Mechanics, Birkhäuser, 2001.

[14] L.A. Peletier, R.K.A.M. Van der Vorst, W.C. Troy, Stationary solutions of a fourth-order nonlinear diffusion equation, Differentsial'nye Uravneniya 31 (2) (1995) 327-337.

[15] D. Smets, J.B. van den Berg, Homoclinic solutions for Swift-Hohenberg and suspension bridges type equations, J. Differential Equations 184 (1) (2002) 78-96.

[16] J.B. Swift, P.C. Hohenberg, Hydrodynamic fluctuations at the convective instability, Phys. Rev. A 51 (1977) 319-328.

[17] J.B. van den Berg, Branches of heteroclinic, homoclinic and periodic solutions in a fourth-order bi-stable system, a numerical study, Master Thesis, Leiden University, October 1996.

[18] J.B. van den Berg, The phase plane picture for a class of fourth order conservative differential equations, J. Differential Equations 161 (2000) 110-153.

[19] G.J.B. van den Berg, L.A. Peletier, W.C. Troy, Global branches of multi-bump periodic solutions of the Swift-Hohenberg equation, Arch. Rational Mech. Anal. 158 (2) (2001) 91-153.

[20] J.B. van den Berg, Dynamics and equilibria of fourth order differential equations, Ph.D. Thesis, Leiden University, December 2000. 